\title{
Nanotheranostics
}

2021; 5(3): 256-274. doi: 10.7150/ntno.51676

Review

\section{Radiolabelling of Extracellular Vesicles for PET and SPECT imaging}

\author{
Azalea A. Khan and Rafael T. M. de Rosales $\bowtie$ \\ Dept. of Imaging Chemistry and Biology, School of Biomedical Engineering and Imaging Sciences, King's College London, St. Thomas' Hospital, London, United \\ Kingdom. \\ $\bowtie$ Corresponding author: Rafael T. M. de Rosales, E-mail: rafael.torres@kcl.ac.uk \\ (C) The author(s). This is an open access article distributed under the terms of the Creative Commons Attribution License (https://creativecommons.org/licenses/by/4.0/). \\ See http://ivyspring.com/terms for full terms and conditions.
}

Received: 2020.08.07; Accepted: 2021.01.09; Published: 2021.02.13

\begin{abstract}
Extracellular vesicles (EVs) such as exosomes and microvesicles have gained recent attention as potential biomarkers of disease as well as nanomedicinal tools, but their behaviour in vivo remains mostly unexplored. In order to gain knowledge of their in vivo biodistribution it is important to develop imaging tools that allow us to track EVs over time and at the whole-body level. Radionuclide-based imaging (PET and SPECT) have properties that allow us to do so efficiently, mostly due to their high sensitivity, imaging signal tissue penetration, and accurate quantification. Furthermore, they can be easily translated from animals to humans. In this review, we summarise and discuss the different studies that have used PET or SPECT to study the behaviour of EVs in vivo. With a focus on the different radiolabelling methods used, we also discuss the advantages and disadvantages of each one, and the challenges of imaging EVs due to their variable stability and heterogeneity.
\end{abstract}

Key words: Exosomes, EVs, Nanomedicine, Imaging, Radiolabelling, PET, SPECT.

\section{Introduction}

Extracellular vesicles (EVs) are cell-derived phospholipid bilayer enclosing vesicles. Once thought to be 'garbage bags' used by cells to excrete unwanted molecules, our current understanding of their function includes cell-to-cell communication and expanded their potential applications to the fields of medical diagnostics and therapy [1-4]. One of the earliest reports on the potential of EVs as therapeutics was in 1996, when EVs were shown to trigger an adaptive immune response [5], but it wasn't until 2007 that the first demonstration that EVs carry functional RNA, and can deliver it to other cells was reported [6]. Since then EVs have been shown to contain a variety of other cell-derived cytosolic molecules, such as proteins, lipids, nucleic acids, among others [7]. Consequently, later research revealed the important role EVs play in cell-cell communication $[4,8]$ and their association in various diseases, such as cancer and metastasis [9-11], neurodegenerative diseases [12, 13], diabetes [14], and inflammatory conditions [15-17]. Recent advances in EV engineering have also demonstrated the possibility of chemically modifying these cell-derived vesicles to improve their therapeutic potential, including the introduction of targeting vectors, stability groups (e.g. PEGylation) as well as the possibility of drug loading, expanding their therapeutic potential even further [18]. For all these reasons, EVs have gained substantial recent attention as potential biomarkers of disease as well as nanotherapeutics.

\section{Classification of EVs}

EVs have been classified in three subtypes, based on their cellular origin as well as their size (Fig. 1): (i) exosomes (30-150 nm), (ii) microvesicles (50-1000 $\mathrm{nm})$, and (iii) apoptotic bodies (500-2000 nm). Exosomes are nanovesicles that are formed by inward budding of the endosomal membrane, and released into the extracellular space by exocytosis [19, 20]. Exosomal membrane is enriched with tetraspanins, such as CD63, CD81 and CD9; as well as endosomal proteins, such as Alix and TSG101 [7]. Unlike 
exosomes, microvesicles are formed by outward budding of the cell membrane with abundant presence of phosphatidylserine in the outer layer of the bilayer [21, 22]. Apoptotic bodies are blebs formed when cells are undergoing apoptosis. However, a recent review outlined that apoptotic bodies are more than just membrane blebs, but are important modulators of immune response [23].

Theoretically, different EVs can be isolated from body fluids or cell culture supernatant by using different (ultra)centrifugation speeds [3], size exclusion chromatography (SEC) or other techniques [24]. However, isolation of individual EV populations is challenging due to their overlap in size and complex physicochemical properties. For example, common exosome biomarkers CD63, CD81 and CD9 have been reported to be present in other $\mathrm{EV}$ sub-groups dependent on the cell type [25, 26]. Hence, adhering to the Minimal Information for Studies of Extracellular Vesicles (MISEV) 2018 guidelines, in this review EVs would be identified by their size [27]. Vesicles of sizes $<200 \mathrm{~nm}$ would be defined as small EVs (sEVs), vesicles in the $200-500 \mathrm{~nm}$ size range would be defined as medium EVs (mEVs), and vesicles $>500 \mathrm{~nm}$ would be defined as large EVs (lEVs).

\section{EVs as nanomedicines}

Of the different subtypes of EVs described above, sEVs or exosomes $(<200 \mathrm{~nm})$ have been explored for their potential as therapeutic nanomedicines [28-30]. The goal of nanomedicine is to improve the therapeutic effects of drugs by improving targeting to disease sites and/or reducing their systemic toxic side effects [31]. Several properties make sEVs an attractive platform for nanomedicine. Compared to other platforms of synthetic origin (e.g. liposomes, polymers), natural EVs are antigen presenting, and depending on their cell of origin may have specific tissue-targeting properties [5, 32, 33]. sEVs can also be engineered to express ligands for better tissue/cell targeting and stability [34, 35], can be used as drug delivery vehicles [36, 37], and have been reported to accumulate in tumours due to the enhanced permeability and retention (EPR) effect [38, 39]. Another important property often cited is their innate ability of crossing the blood-brain barrier, which is a significant biological barrier for other nanomedicinal drug delivery platforms [40-42]. sEVs from immune cells may have therapeutic properties by themselves. For example, neutrophil-derived sEVs have been shown to induce inflammatory resolution [43], while sEVs containing foreign antigens/molecules have the ability to trick the immune system, as is the case for many viral diseases [44]. Recent reports have also shown that sEVs can be more efficient as gene therapy and drug delivery vehicles than systems based on liposomes [45-47]. All these exciting results have resulted in many EV-based therapies progressing quickly towards clinical trials, with over 15 trials registered on $\mathrm{NIH}$ ClinicalTrials.gov, including 3 studies looking into treatment for COVID-19, and successful completion of six studies [48-53]. However, there is still a lack of information regarding their fate in vivo after administration, particularly when these investigational therapeutic nanomedicines are used in humans.

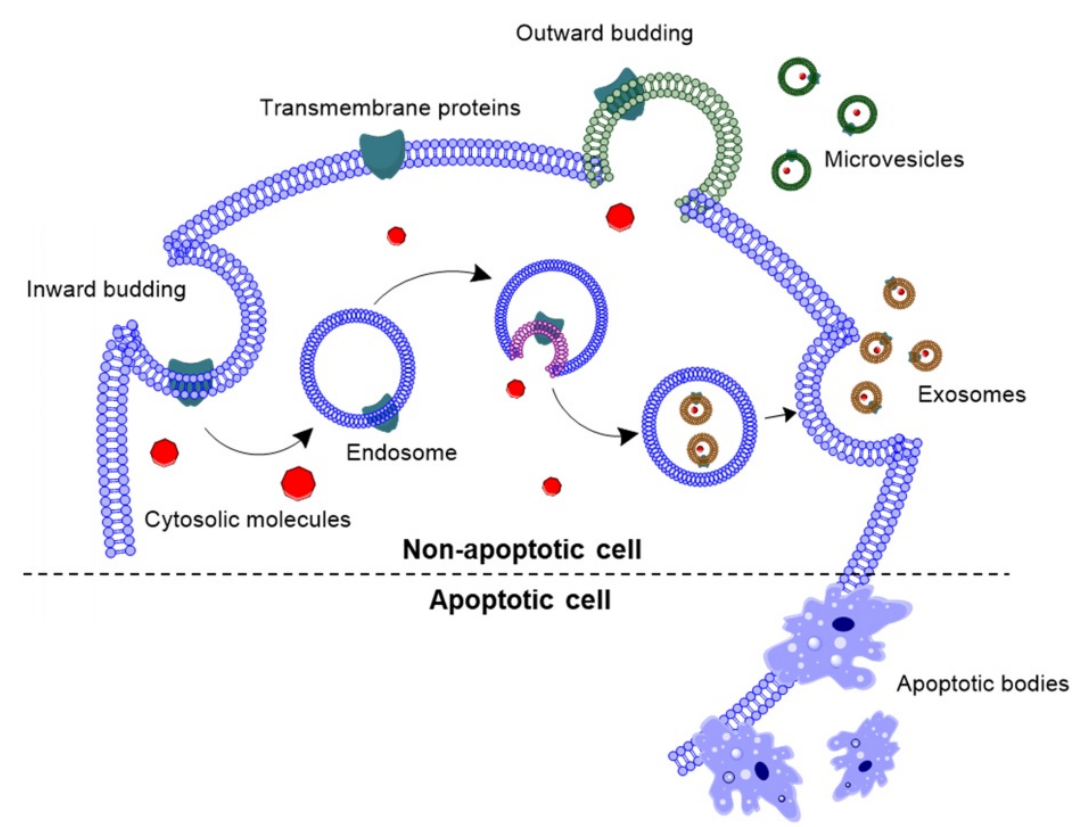

Figure 1. Biogenesis of extracellular vesicles. Exosomes are formed by inward budding of the endosomal membrane, followed by being released into the extracellular space. Whereas, microvesicles are formed by outward budding of the cell membrane, and apoptotic bodies are formed by outward blebbing of apoptotic cell membrane. 
A

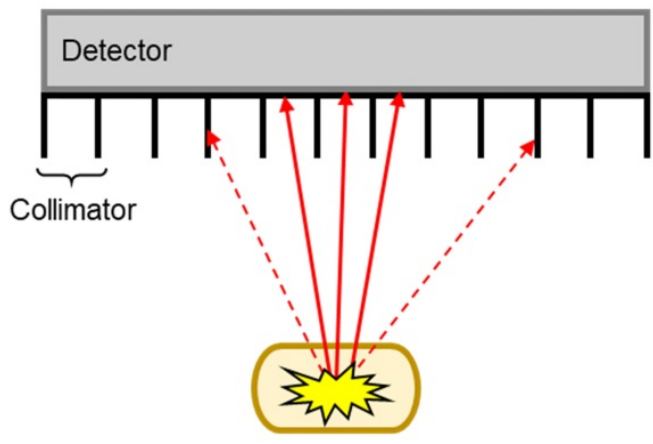

B

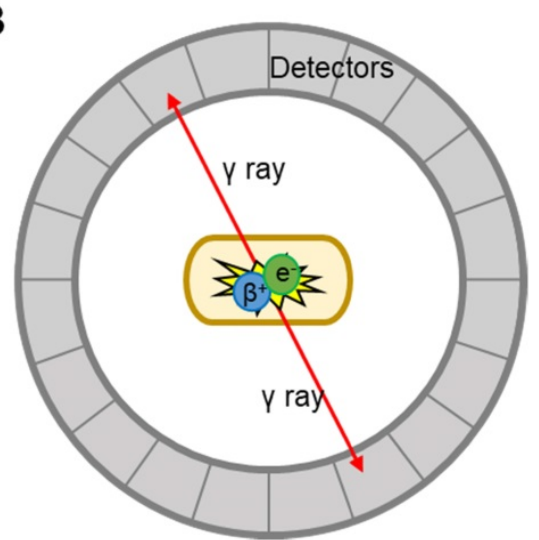

Figure 2. Schematic representation of SPECT and PET detection. A) Linear SPECT detector with collimators; dotted arrows = photons that are absorbed by the collimators, solid arrows = photons that reach the detector. B) Circular ring of PET detectors detect photons arriving simultaneously in opposite directions.

To tackle this lack of information and efficiently develop EV-based therapies, it is important to integrate imaging-based in vivo tracking techniques early in the developmental process, to help answer questions regarding their fate after administration into patients/subjects. This will facilitate not only their development but also the progress of clinical trials, allowing the early identification of the most suitable candidates to take forward. As a developmental tool, non-invasive imaging of EV therapies would be an ideal method to monitor and quantify EV biodistribution over time and enable elucidation of their pharmacokinetic profiles. Furthermore, taking into account patient and disease heterogeneity, integrating imaging with EV-based therapies could provide a tool to identify patients/lesions that are likely to respond to the treatment. Such personalised medicine approach would allow optimisation of treatment strategies to suit individual patients' needs [54].

\section{Imaging EVs in vivo}

Of the several imaging techniques that have been utilised to track EV biodistribution over time, optical imaging (OI) is the most popular for its simplicity, and for being both cost and time effective. For these reasons, it has been used in many studies to understand and characterise EV properties [55]. Nonetheless, OI suffers from inherent physical limitations, mainly poor tissue depth penetration hence its application is limited to the preclinical setting (for whole-body animal imaging), clinical intraoperative and in vitro/ex vivo imaging. Other imaging techniques don't suffer from this limitation, particularly computed tomography (CT), magnetic resonance imaging (MRI), and the nuclear medicine techniques - single photon emission computed tomography (SPECT) and positron emission tomography (PET). All of these allow whole-body imaging with unlimited imaging signal depth penetration [56]. Furthermore, they are available in both the preclinical and clinical setting.

MRI and CT benefit from excellent spatiotemporal resolution but suffer from low sensitivity (i.e. large amount of contrast agent required to allow detection). This is a major limitation for EV imaging, as the concentration of contrast agent per EV required for efficient imaging signal can be potentially damaging to the vesicles. This is perhaps one of the reasons behind the low number of studies that used MRI for EV imaging [57-62]. CT has only been used in combination with nuclear imaging techniques (SPECT or PET) for EV imaging. These two techniques offer higher sensitivity compared to CT/MRI (ca. 106-fold), no background signal, and allow whole body imaging with accurate signal quantification. By radiolabelling EVs with appropriate radioactive isotopes, or radionuclides, it is possible to track/image EVs using either SPECT or PET. The main differences between SPECT and PET imaging lies in the type of radioisotope used, and how the signal is detected and converted into 3D images (Fig. 2). SPECT detect radionuclides that decay by emission of $y$ photons. These $y$ photons have definite

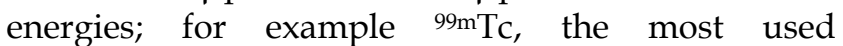
radionuclide in nuclear medicine with a decay half-life $\left(t_{1 / 2}\right)$ of $6 \mathrm{~h}$, emits $140 \mathrm{keV}$ y photons. These radionuclides are detected by a rotating gamma camera to generate a 3D image. Collimators are used to only allow radiation at certain angles to reach the detector and thus determine the source (Fig. 2A) [63]. PET, on the other hand, uses radionuclides that decay by positron $\left(\beta^{+}\right)$emission. Positrons travel for a short distance (depending on their energy, sub-mm to several $\mathrm{mm}$ ) before annihilating with an electron, an event that produces two $\gamma$ rays of equal energy (511 $\mathrm{keV})$ simultaneously in opposite directions. These $\gamma$ photons are detected by a circular ring of detectors 
that maps out simultaneously arriving $\gamma$ rays at $180^{\circ}$, a technique known as coincidence detection (Fig. 2B) [64]. These differences in detection techniques and instrumentation lead to differences in image quality and sensitivity. In general terms, clinical PET is superior to SPECT in some aspects as outlined in Table 1 below. SPECT, however, has the major advantage that allows multi-radionuclide imaging with radionuclides that emit $\gamma$ rays of different energies.

In this review we focus on the different methods explored to date to radiolabel EVs that allow in vivo tracking with both PET and SPECT imaging. We critically review the main advantages and disadvantages of each method, and where possible discuss them into the context of their in vivo imaging properties. For reviews on imaging EVs using other imaging techniques, we point the reader to excellent publications available [41, 55, 66, 67]. A total of 16 published articles and pre-prints that included radiolabelling of EVs and EV-mimetics were found (July 2020, see Supporting Information for methodology) and analysed in this review, of which 11 were on SPECT imaging and 5 of them on PET imaging. These articles were further classified into the different types of radiolabelling methods discussed below (Fig. 3).

\section{Radiolabelling of EVs}

Due to the similarities in their physical structures, the different chemical concepts that allow radiolabelling of liposomes can also be applied to EVs [68]. Specifically, both EVs and liposomes are similar in size and consist of a phospholipid bilayer enclosing an aqueous core capable of carrying a chemical cargo. A recent review by Man et al. outlines the different methods that have been employed to radiolabel and image liposomal systems to date, including their pros and cons [69]. Similar to liposomes, there are also two primary methods for radiolabelling EVs - surface and

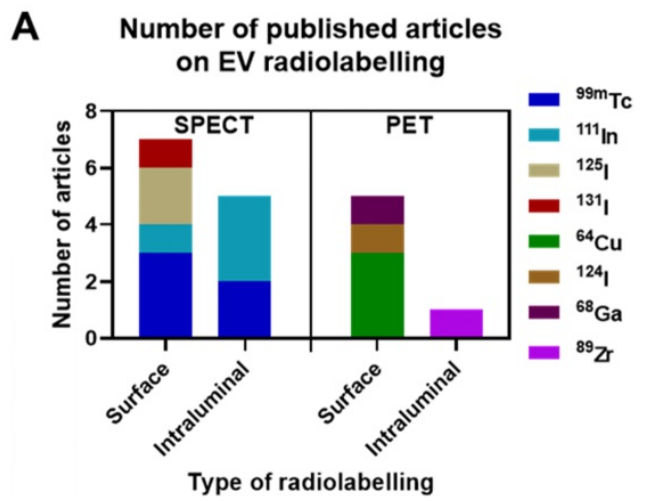

intraluminal - that we will briefly describe below (Fig. 4).

\section{Surface radiolabelling}

Surface or membrane radiolabelling is the most common method to radiolabel EVs. This involves incorporation of the radionuclide directly into the lipid membrane or attached to the membrane proteins, either directly or via covalent chemical bond formation. Four main methods have been applied to achieve this: 1) genetic modification, 2) direct incorporation of radionuclide into the membrane, 3 ) radionuclide attachment via a chelator (i.e. radiometal-binding chemical group) on the surface, and 4) direct incorporation of radionuclide into membrane proteins (Fig. 4A). Direct incorporation of radionuclides relies on non-specific affinities between the radionuclides and the EV membrane components. For the surface chelation methods the radiotracer is conjugated to the membrane, typically, via surface amine groups using standard bioconjugate chemistry techniques. More detailed descriptions behind these methods are described in the 'Radiolabelling of EVs using SPECT radionuclides' and 'Radiolabelling of EVs using SPECT radionuclides' sections below with the corresponding radiotracers (vide infra). A potential limitation of surface radiolabelling methods in general is that they could compromise the integrity of the EV's surface, particularly if they involve chemical modification of membrane proteins, or substantial modification of the membrane composition. The importance of EV surface proteins and lipids in their behaviour has been demonstrated in various studies $[32,70-74]$, and a recently published review described the different methods to modify the surface of EVs [75]. These alterations are usually applied to understand EV function or to improve tissue targeting; hence those aimed at enabling radiolabelling have significant potential to alter their natural physicochemical properties and biodistribution.

\section{B Percentage of EV radiolabelling based on different methods}

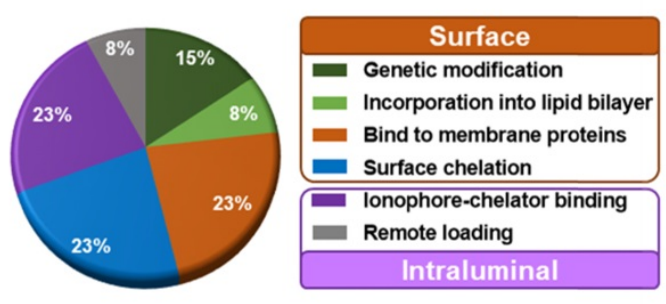

Figure 3. Research articles published until July 2020. A) EV radiolabelling with various SPECT and PET radionuclides employing either surface or intraluminal radiolabelling strategy. B) Percentage of publications using different techniques to achieve surface and intraluminal radiolabelling. Please refer to the supplementary information for methodology. 
A

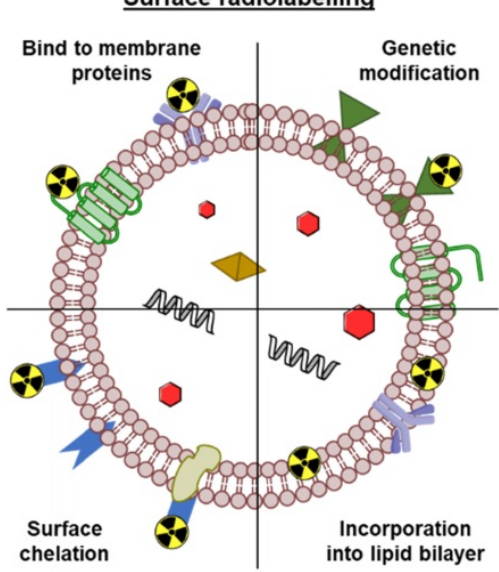

B Intraluminal radiolabelling

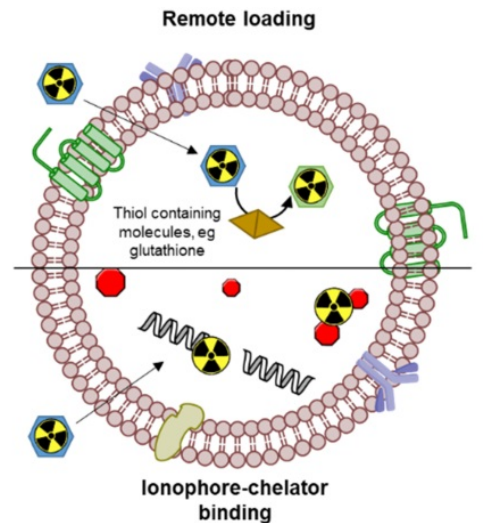

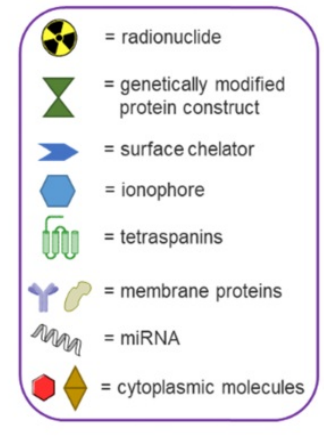

Figure 4. Schematic representation of different EV radiolabelling methods. A) Surface radiolabelling: radionuclide can be incorporated into the EV membrane directly or via a chelator. B) Intraluminal radiolabelling: ionophores allow radionuclides to be transported across the lipid membrane where they can be trapped as their lipophilicity changes or bind to metal chelating biomolecules.

Table 1. Differences in clinical SPECT and PET imaging. Data in the table is collated from Rahmim et al. [65] and James et al. [56].

\begin{tabular}{|c|c|c|c|c|c|}
\hline & Detection method & $\begin{array}{l}\text { Geometric efficiency } \\
\text { (percentage of detected to emitted } \gamma \text { rays) }\end{array}$ & Temporal resolution & Spatial resolution & $\begin{array}{l}\text { Sensitivity } \\
\text { (concentration of radiotracer needed) }\end{array}$ \\
\hline SPECT & Collimator detection & $\sim 0.01 \%$ & Minutes & $8-10 \mathrm{~mm}$ & $10^{-10}$ to $10^{-11} \mathrm{M}$ \\
\hline PET & Coincidence detection & $\sim 1 \%$ & Seconds - minutes & $5-7 \mathrm{~mm}$ & $10^{-11}$ to $10^{-12} \mathrm{M}$ \\
\hline
\end{tabular}

\section{Intraluminal radiolabelling}

An alternative approach to radiolabel EVs is to entrap the radiotracer inside the intra-vesicular space (Fig. 4B). Thus, the lipid bilayer membrane is expected to protect the radionuclide from trans-chelation by extra-vesicular components, such as serum proteins. This is in contrast to surface radiolabelling, where the radionuclide is more exposed to extra-EV trans-chelation. To radiolabel EVs intraluminally, the radionuclide needs to cross the lipid bilayer and stay within the EV. To achieve this, two methods have been explored to date: 1) remote loading, and 2) ionophore-chelator binding. The first method takes advantage of endogenous intravesicular glutathione that is capable of transforming some complexes, such as [99mTc]Tc-hexamethylpropyleneamine oxime ([99mTc]Tc-HMPAO), from lipophilic to hydrophilic [76]. Once the lipophilic radiotracer complex passes through the lipid bilayer membrane, it is converted into its hydrophilic form and thus, gets trapped inside the aqueous core of the EV. The ionophore-chelator binding method exploits well-known ionophore ligands, such as tropolone and 8-hydroxyquinolin (oxine), that form a metastable and neutral complex with the radiometals, allowing them to be transported across the lipid membrane. This method is commonly used to radiolabel cells as well as liposomal nanomedicines, where the radiometal can bind to metal chelating moieties within the liposomal cargo
[77]. In the case of EVs, the radiometal is expected to bind to intravesicular proteins and/or nucleic acids. The main disadvantage of intraluminal radiolabelling approaches, especially those based on ionophores, is that we lack the knowledge of exactly which component of the EVs' intraluminal space the radionuclide binds to. This may complicate interpretation of in vivo images, particularly at late timepoints when EV lipid bilayer fragmentation may be significant and due to the fact that some radionuclides - particularly radiometals such as ${ }^{64} \mathrm{Cu}$ - that accumulate in the same organs as EVs (e.g. liver, spleen) [69].

The two main radiolabelling categories described above, surface and intraluminal, have been employed with both SPECT and PET radionuclides with various degrees of radiolabelling capabilities. In the next sections we will describe the different EV radiolabelling studies reported to date, that we have classified in to SPECT radionuclides (Table 2) and PET radionuclides (Table 3 ).

\section{Radiolabelling of EVs using SPECT radionuclides}

The first report reporting radiolabelling of sEVs was by Morishita et al. in 2014 (publication in 2015, but available online from Nov 2014) [78]. Using plasmid transfection, lactadherin - a glycoprotein found on cell membrane - was replaced with streptavidin on B16-BL6 cells and cultured for sEV 
isolation. Streptavidin containing B16-BL6 sEVs (70 \pm $3 \mathrm{~nm}$ ) were radiolabelled with biotin-conjugated ${ }^{125} \mathrm{I}$ $\left(t_{1 / 2}=59.4 \mathrm{~d}\right),\left[{ }^{125} \mathrm{I}\right] \mathrm{I}-\mathrm{IBB}$. Strong binding affinity $\left(\mathrm{K}_{\mathrm{d}}\right.$ $\left.\sim 10^{15} \mathrm{M}^{-1}\right)$ makes the streptavidin-biotin conjugate a popular pretargeting method in radioimmunotherapy [94]. The radiolabelling yield (RLY) of B16-BL6 sEVs with [125I]I-IBB was $\sim 80 \%$, and in vitro serum stability was $\sim 95 \%$ for up to $4 \mathrm{~h}$. Despite blood clearance of ${ }^{125}$ I-labelled B16-BL6 sEVs having the same profile as the free radiotracer ([125I]I-IBB) in healthy mice (Fig. 5A), their ex vivo biodistributions were significantly different with 125I-labelled B16-BL6 sEVs showing accumulation in liver, lung and spleen (Fig. 5B). Surprisingly, ${ }^{125} \mathrm{I}$ uptake in the thyroid was not mentioned in the paper. Thyroid is an organ of interest when using iodine as a sign of radiochemical instability with iodinated radiotracers, due to the presence of the human $\mathrm{Na}^{+} / \mathrm{I}^{-}$symporter (hNIS). Stomach uptake, another organ with high affinity for free iodide via hNIS was very low, suggesting in vivo radiochemical stability during the $4 \mathrm{~h}$ study. This study, however, is the only study to carry out mathematical pharmacokinetic modelling of radiolabelled sEVs. The same group later published another study based on the same radiolabelling method [79], and reported higher retention of sEVs in xenograft tumours of up to $200 \mathrm{~mm}^{3}$ volume compared to larger tumours of up to $500 \mathrm{~mm}^{3}$ when injected intratumorally (Fig. 5C).

Although useful as a preclinical tool, genetic modification is a challenge in terms of clinical translation. Radiolabelling with iodine can easily be performed using iodination beads (iodo-bead method), which consists of a polystyrene bead coated with an oxidising agent facilitating the reduction of tyrosine residues and iodine substitution [95], thus iodinating EV surface proteins. Using this method, Rashid et al. achieved $>80 \%$ RLY with ${ }^{131}$ I $\left(t_{1 / 2}=8 \mathrm{~d}\right)$ for sEVs derived from $4 \mathrm{~T} 1$ cells [80]. Although up to $80 \%$ of the radiolabelled sEVs were stable in serum for $24 \mathrm{~h}$; in vivo imaging at early time-points (ca. $3 \mathrm{~h}$ ) showed high thyroid, stomach and bladder uptake for both tumour cell- and healthy cell-derived sEVs, which correlates with release of free 131I (Fig. 5D). It has been previously reported that radio-iodination using the iodo-bead method is prone to rapid deiodination in vivo as early as $2 \mathrm{~h}$ post injection [96]. Hence, it seems that sEVs radiolabelled using the iodo-bead method suffer from low in vivo radiochemical stability and cannot reliably be used to determine their biodistribution.

Table 2. Summary of reports of EV radiolabelling with SPECT radioisotopes. The hydrodynamic size of EVs are stated for unmodified EVs before radiolabelling, as appropriate. Radiolabelling condition column shows EV and radiotracer incubation time, temperature, and the amount of EVs used per reaction. Data shown as reported by the authors. RLY $=$ radiolabelling yield, $\mathrm{UC}=$ ultracentrifugation, $\mathrm{UF}=$ ultrafiltration, $\mathrm{SEC}=$ size exclusion chromatography, RT = room temperature, iTLC = instant thin layer chromatography; $*$ data taken from the figures.

\begin{tabular}{|c|c|c|c|c|c|c|c|c|}
\hline Type & Radionuclide & $\begin{array}{l}\text { Source of sEVs / EMVs; } \\
\text { size; isolation method }\end{array}$ & $\begin{array}{l}\text { Radiolabelling } \\
\text { condition }\end{array}$ & Purification & RLY & $\begin{array}{l}\text { In vitro stability; } \\
\text { assessed by }\end{array}$ & $\begin{array}{l}\text { In vivo } \\
\text { imaging }\end{array}$ & Ref. \\
\hline \multirow[t]{7}{*}{$\begin{array}{l}\text { Surface } \\
\text { radiolabelling }\end{array}$} & 125I-biotin & $\begin{array}{l}\text { B16-BL6 mouse melanoma } \\
\text { cells; } 70 \pm 3 \mathrm{~nm} \text {; UC }\end{array}$ & $\begin{array}{l}30 \mathrm{~min} ; 37^{\circ} \mathrm{C} ; \\
10 \mu \mathrm{g}\end{array}$ & Not reported & $\sim 80 \% *$ & $\begin{array}{l}>95 \% \text { serum stability at } 4 \\
\text { h; UF }\end{array}$ & $x$ & [78] \\
\hline & & & As above & & & & $x$ & [79] \\
\hline & $\begin{array}{l}\mathrm{Na}^{131} \mathbf{I}+ \\
\text { iodo-bead } \\
\text { method }\end{array}$ & Various & $\begin{array}{l}30 \text { min; RT; } \\
\text { not reported }\end{array}$ & $\begin{array}{l}\text { UF = Nanosep } \\
\text { 100k Omega }\end{array}$ & $\begin{array}{l}>80 \% \text { for } \\
4 \mathrm{~T} 1 \mathrm{EVs}^{*}\end{array}$ & $\begin{array}{l}\text { ca. } 80 \% \text { serum stability at } \\
24 \mathrm{~h}^{*} \text {; iTLC }\end{array}$ & $\checkmark$ & [80] \\
\hline & 99mTc-tricarbonyl & $\begin{array}{l}\text { Human red blood cells; } 188 \\
\pm 11 \mathrm{~nm} ; \mathrm{UC}+\mathrm{UF}+\mathrm{SEC}\end{array}$ & $\begin{array}{l}30 \mathrm{~min} ; \mathrm{RT} \\
0.6 \mathrm{~mL}\end{array}$ & $\begin{array}{l}\text { Zeba spin } \\
\text { desalting column }\end{array}$ & $\begin{array}{l}38.8 \pm \\
6.2 \%\end{array}$ & No data given & $\checkmark$ & [81] \\
\hline & ${ }^{99 \mathrm{~m} T \mathrm{Tc}-\text { tricarbonyl }}$ & $\begin{array}{l}\text { HEK } 293 T \text { human } \\
\text { embryonic kidney cells; } \sim 77 \\
\text { nm; not reported }\end{array}$ & $\begin{array}{l}1 \mathrm{~h} ; 37^{\circ} \mathrm{C} ; \\
20 \mu \mathrm{g}\end{array}$ & None used & $>98 \%$ & $\begin{array}{l}96 \% \text { saline stability at } 24 \\
\text { h; TLC }\end{array}$ & $\checkmark$ & [82] \\
\hline & $\begin{array}{l}\text { 99mTc } \\
\left(+\mathrm{SnCl}_{2}\right)\end{array}$ & $\begin{array}{l}\text { Goat milk; } 122 \pm 1 \mathrm{~nm} \text {; UC } \\
\text { and SEC }\end{array}$ & $\begin{array}{l}30 \mathrm{~min} ; 37^{\circ} \mathrm{C} ; \\
75 \mu \mathrm{g}\end{array}$ & $\begin{array}{l}\text { Exosome spin } \\
\text { column }\end{array}$ & $37 \pm 9 \%$ & $\begin{array}{l}\text { 95\% PBS stability at } 48 \mathrm{~h} \text {; } \\
\text { iTLC }\end{array}$ & $\checkmark$ & [83] \\
\hline & 111In-DTPA & $\begin{array}{l}\text { B16-F10 mouse melanoma } \\
\text { cells; } 132 \pm 6 \mathrm{~nm} \text {; UC }\end{array}$ & $\begin{array}{l}30 \mathrm{~min} ; 37^{\circ} \mathrm{C} ; \\
1 \times 10^{11} \mathrm{sEVs}\end{array}$ & $\begin{array}{l}\text { SEC }=\text { Sepharose } \\
\text { CL-2B }\end{array}$ & $\begin{array}{l}19.2 \pm \\
4.5 \%\end{array}$ & $\begin{array}{l}86.8 \pm 3.1 \% \text { PBS stability, } \\
\text { and } 80.4 \pm 1.6 \% \text { serum } \\
\text { stability at } 24 \mathrm{~h} \text {; iTLC }\end{array}$ & $\checkmark$ & [84] \\
\hline \multirow[t]{6}{*}{$\begin{array}{l}\text { Intraluminal } \\
\text { radiolabelling }\end{array}$} & ${ }^{111}$ In-oxinate & $\begin{array}{l}\text { PC3 human prostate cancer } \\
\text { cells; } 140 \pm 59 \mathrm{~nm} \text {; UC }\end{array}$ & $\begin{array}{l}20 \mathrm{~min} ; \mathrm{RT} ; \\
2.5-3.7 \mathrm{mg} / \mathrm{mL}\end{array}$ & SEC $=$ P6 column & $81 \%$ & No data given & $x$ & [85] \\
\hline & & $\begin{array}{l}\text { MCF-7 human breast cancer } \\
\text { cells; } 130 \pm 57 \mathrm{~nm} \text {; UC }\end{array}$ & & & $67 \%$ & & & \\
\hline & 111In-oxinate & $\begin{array}{l}\text { HEK } 293 \text { human embryonic } \\
\text { kidney cells; } 106 \pm 14 \text { nm; } \\
\text { UF + UC }\end{array}$ & $\begin{array}{l}30 \text { min; RT; } \\
1 \times 10^{9} \mathrm{sEVs} / \mathrm{mL}\end{array}$ & $\begin{array}{l}\text { UF }=\text { Amicon ultra } \\
100 \mathrm{kDa}\end{array}$ & $>98 \%$ & $\begin{array}{l}>92 \% \text { serum stability at } \\
24 \mathrm{~h} \text {; iTLC }\end{array}$ & $\checkmark$ & [86] \\
\hline & ${ }^{111}$ In-tropolone & $\begin{array}{l}\text { B16-F10 mouse melanoma } \\
\text { cells; } 132 \pm 6 \mathrm{~nm} \text {; UC }\end{array}$ & $\begin{array}{l}20 \mathrm{~min} ; 37^{\circ} \mathrm{C} \\
1 \times 10^{11} \mathrm{sEVs}\end{array}$ & $\begin{array}{l}\text { SEC }=\text { Sepharose } \\
\text { CL-2B }\end{array}$ & $\begin{array}{l}4.7 \pm \\
0.4 \%\end{array}$ & $\begin{array}{l}43.4 \pm 10.1 \% \text { PBS stability, } \\
\text { and } 14.2 \pm 2.8 \% \text { serum } \\
\text { stability at } 24 \text { h; SEC }\end{array}$ & $\checkmark$ & [84] \\
\hline & 99mTc-HMPAO & $\begin{array}{l}\text { Raw } 264.7 \text { mouse } \\
\text { macrophages; } 218 \pm 8 \mathrm{~nm} \text {; } \\
\text { serial extrusion }\end{array}$ & $\begin{array}{l}1 \mathrm{~h} ; \mathrm{RT} \\
100 \mu \mathrm{g}\end{array}$ & $\begin{array}{l}\text { SEC }=\text { PD-10, } \\
\text { MW3000 spin } \\
\text { column }\end{array}$ & $>93 \%$ & $\begin{array}{l}\sim 90 \% \text { serum stability at } 5 \\
\mathrm{~h} ; \text { iTLC }\end{array}$ & $\checkmark$ & [87] \\
\hline & $\begin{array}{l}{ }^{99 \mathrm{~m} T \mathrm{Tc}} \\
\left(+\mathrm{SnCl}_{2}\right)\end{array}$ & $\begin{array}{l}\text { Rat red blood cells; } 201 \pm 16 \\
\mathrm{~nm} \text {; serial extrusion }\end{array}$ & $\begin{array}{l}20 \mathrm{~min} ; 37^{\circ} \mathrm{C} ; \\
100 \mu \mathrm{g}\end{array}$ & $\begin{array}{l}\text { UC (only if RLY }< \\
95 \% \text { on iTLC) }\end{array}$ & $100 \%$ & $\begin{array}{l}93 \pm 3 \% \text { serum stability at } \\
24 \mathrm{~h} \text {; iTLC }\end{array}$ & $\checkmark$ & [88] \\
\hline
\end{tabular}


Table 3. Summary of reports of EV radiolabelling with PET radioisotopes. The hydrodynamic size of EVs are stated for unmodified EVs before radiolabelling, as appropriate. Radiolabelling conditions column shows EV and radiotracer co-incubation time, temperature, and the amount of EVs used per reaction. Data shown as reported by the authors. RLY = radiolabelling yield, $U C=$ ultracentrifugation, $S E C=$ size exclusion chromatography, RT = room temperature, iTLC = instant thin layer chromatography; * data taken from the figures.

\begin{tabular}{|c|c|c|c|c|c|c|c|c|}
\hline Type & Radionuclide & $\begin{array}{l}\text { Source of sEVs; size; } \\
\text { isolation method }\end{array}$ & $\begin{array}{l}\text { Radiolabelling } \\
\text { conditions }\end{array}$ & Purification & RLY & $\begin{array}{l}\text { In vitro stability; } \\
\text { assessed by }\end{array}$ & $\begin{array}{l}\text { In vivo } \\
\text { imaging }\end{array}$ & Ref. \\
\hline \multirow[t]{5}{*}{$\begin{array}{l}\text { Surface } \\
\text { radiolabelling }\end{array}$} & $\begin{array}{l}\mathrm{Na}^{124} \mathrm{I}+\text { iodogen } \\
\text { method }\end{array}$ & $\begin{array}{l}\text { MLP29 mouse liver cells; 130 } \\
\text { nm; UC }\end{array}$ & $\begin{array}{l}2 \mathrm{~h} ; 25^{\circ} \mathrm{C} \\
0.4 \mu \mathrm{g}\end{array}$ & $\begin{array}{l}\text { SEC }= \\
\text { Sephadex G25 } \\
\text { (DNA grade) }\end{array}$ & $\begin{array}{l}\text { Glycosylated =17 } \\
\pm 2 \% \\
\text { Non-glycosylated } \\
=19 \pm 1 \%\end{array}$ & $\begin{array}{l}>90 \% \text { PBS stability at } \\
72 \text { h; iTLC }\end{array}$ & $\checkmark$ & [89] \\
\hline & ${ }^{64} \mathrm{Cu}$-DOTA & $\begin{array}{l}\text { Human umbilical cord blood } \\
\text { mononuclear cells; } \sim 110 \mathrm{~nm} \text {; } \\
\text { UC }\end{array}$ & $\begin{array}{l}1 \mathrm{~h} ; \mathrm{RT} \\
>300 \mu \mathrm{g}\end{array}$ & $\begin{array}{l}\text { MW3000 } \\
\text { exosome spin } \\
\text { column }\end{array}$ & $16-25 \%$ & $\begin{array}{l}94 \% \text { serum stability at } \\
24 \mathrm{~h}, 95 \% \text { blood } \\
\text { stability at } 1 \mathrm{~h} \text {; iTLC }\end{array}$ & $\checkmark$ & [90] \\
\hline & ${ }^{64} \mathrm{Cu}-\mathrm{NOTA}$ & $\begin{array}{l}4 \mathrm{~T} 1 \text { mouse breast cancer cells; } \\
106.3 \pm 0.3 \mathrm{~nm} \text { (volume } \\
\text { weighted); UC }\end{array}$ & $\begin{array}{l}30 \mathrm{~min} ; 37^{\circ} \mathrm{C} \\
300 \mu \mathrm{g}\end{array}$ & $\mathrm{SEC}=\mathrm{PD}-10$ & $\begin{array}{l}\text { Non-PEGylated }= \\
91.2 \pm 0.2 \% \\
\text { PEGylated }=85.7 \pm \\
0.7 \%\end{array}$ & $\begin{array}{l}\text { Non-PEGylated }=80.4 \\
\pm 1.3 \% \text { PEGylated }= \\
95.7 \pm 0.9 \% \text { serum } \\
\text { stability at } 24 \mathrm{~h} ; \text { iTLC }\end{array}$ & $\checkmark$ & [91] \\
\hline & ${ }^{64} \mathrm{Cu}-\mathrm{NOTA}-\mathrm{Cy} 7$ & $\begin{array}{l}\text { 4T1 mouse breast cancer cells; } \\
\sim 100 \text { nm; ExoQuick }{ }^{\circledR}\end{array}$ & $\begin{array}{l}5 \mathrm{~min} ; 37^{\circ} \mathrm{C} ; 100 \\
\mu \mathrm{g}\end{array}$ & ExoQuick ${ }^{\circledR}$ & $\sim 98 \%$ & $\begin{array}{l}>95 \% \text { serum stability } \\
\text { at } 36 \mathrm{~h}^{*} \text {; iTLC }\end{array}$ & $\checkmark$ & [92] \\
\hline & ${ }^{68 \mathrm{Ga}-N O T A-C y 7}$ & & $\begin{array}{l}30 \mathrm{~min} ; 25^{\circ} \mathrm{C} ; \\
100 \mu \mathrm{g}\end{array}$ & & Not reported & Not reported & & \\
\hline \multirow[t]{3}{*}{$\begin{array}{l}\text { Intraluminal } \\
\text { radiolabelling }\end{array}$} & ${ }^{89} \mathrm{Zr}$-oxinate & $\begin{array}{l}\text { B16-F10.GFP mouse } \\
\text { melanoma cells; } 146 \pm 2 \text { nm; } \\
\text { ExoQuick }^{\circledR}\end{array}$ & $\begin{array}{l}1 \mathrm{~h} ; 37^{\circ} \mathrm{C} ; \\
1 \times 10^{10} \mathrm{sEVs}\end{array}$ & $\begin{array}{l}\text { MW3000 } \\
\text { exosome spin } \\
\text { column }\end{array}$ & $6 \pm 1 \%$ & Not reported & $x$ & [93] \\
\hline & & $\begin{array}{l}\text { MDA-MB-231.CD63-GFP } \\
\text { human breast cancer cells; } 121 \\
\pm 14 \mathrm{~nm} \text {; UC }\end{array}$ & $\begin{array}{l}20 \mathrm{~min} ; 37^{\circ} \mathrm{C} \\
1 \times 10^{10} \mathrm{sEVs}\end{array}$ & $\begin{array}{l}\text { SEC }= \\
\text { Sepharose } \\
\text { CL-2B }\end{array}$ & $6 \pm 1 \%$ & & $x$ & \\
\hline & & $\begin{array}{l}\text { PANC1 human pancreatic } \\
\text { cancer cells; } 97 \pm 4 \mathrm{~nm} \text {; UC }\end{array}$ & $\begin{array}{l}20 \mathrm{~min} ; 37^{\circ} \mathrm{C} \\
1 \times 10^{11} \mathrm{sEVs}\end{array}$ & & $23 \pm 7 \%$ & $\begin{array}{l}76 \pm 3 \% \text { PBS stability at } \\
26 \mathrm{~h} ; \text { iTLC }\left(37^{\circ} \mathrm{C}\right)\end{array}$ & $\checkmark$ & \\
\hline
\end{tabular}

The long half-lives of radioiodines are ideal for

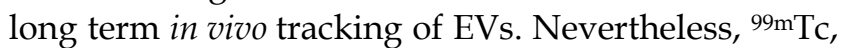
with a shorter half-life of $6 \mathrm{~h}$, is the most commonly used radionuclide for imaging of EVs probably due the availability/low cost and favourable radiation properties of this radionuclide for imaging. The first use of ${ }^{99 \mathrm{~m} T c-l a b e l l e d ~ E V s}$ was reported by Varga et al.,

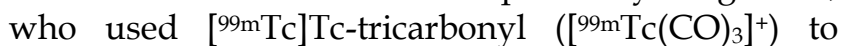
label red blood cell- (RBC) derived sEVs $(188 \pm 11 \mathrm{~nm})$ [81]. Unlike liposomes, that lack appropriate donor ligands and require surface modification [97], EVs should be able to bind to $\left[{ }^{99} \mathrm{~m} \mathrm{Tc}(\mathrm{CO})_{3}\right]^{+}$via surface proteins, most likely involving histidine donors [98]. A RLY of $38.8 \pm 6.2 \%$ was achieved after $30 \mathrm{~min}$ incubation at room temperature, a relatively low RLY which is not surprising as efficient binding to this inert complex requires high temperatures incompatible with biomolecules [99]. An in vivo imaging comparison between $\left[{ }^{99 \mathrm{~m}} \mathrm{Tc}(\mathrm{CO})_{3}\right]^{+}$and $\left[{ }^{99 \mathrm{mTc}}(\mathrm{CO})_{3}\right]^{+}-\mathrm{RBC}-\mathrm{sEVs}$ is consistent with efficient radiolabelling and high stability for the latter. However, the short imaging timeframe of the study $(<$ $2 \mathrm{~h}$ ) does not allow evaluation of the long-term in vivo stability of $\left[{ }^{99 \mathrm{~m}} \mathrm{Tc}(\mathrm{CO})_{3}\right]^{+}-\mathrm{RBC}-\mathrm{sEVs}$ (Fig. 6A-B). A more recent report also exploited the use of $\left[{ }^{99 \mathrm{~m} \mathrm{Tc}}(\mathrm{CO})_{3}\right]^{+}$to radiolabel human epidermal growth factor receptor 2 (HER2) targeted- HEK 293T sEVs [82]. Contrary to the previous study, a high RLY was achieved by incubation of $\left[{ }^{99 \mathrm{~m}} \mathrm{Tc}(\mathrm{CO})_{3}\right]^{+}$with the sEVs at a relatively low temperature of $37^{\circ} \mathrm{C}$ for $60 \mathrm{~min}$. In vivo imaging showed expected high liver uptake, but no spleen, as well as significant kidney and intestine signal that could potentially indicate free $\left.{ }^{[99 \mathrm{~m}} \mathrm{Tc}(\mathrm{CO})_{3}\right]^{+}$.

An alternative surface radiolabelling method with $99 \mathrm{mTc}$ involves reduction of the unreactive

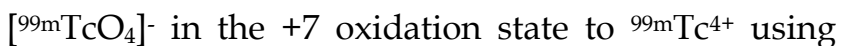
stannous chloride $\left(\mathrm{SnCl}_{2}\right)$, a commonly used $\mathrm{RBC}$ radiolabelling method [100]. Gonzalez et al. optimised this method for sEV radiolabelling and achieved $37 \pm$ 9\% RLY using $2 \mathrm{mM} \mathrm{SnCl}_{2}$ and $75 \mu \mathrm{g}$ of milk-derived sEVs $(122 \pm 1 \mathrm{~nm})$ [83]. Using this method, ${ }^{99 \mathrm{mTc}}$ was incorporated directly into the milk-derived sEV membrane, although the exact binding site of the ${ }^{99} \mathrm{~m}^{4+}$ ion is unclear and is likely to be non-specific binding to surface proteins. In vitro stability within 48 $\mathrm{h}$ in PBS was high, although it would have been interesting to test with more challenging conditions (e.g. in the presence of serum components). Looking into different administration routes the authors noted significant differences in the biodistribution depending on the injection route, with mainly liver and spleen uptake via intravenous (iv.) administration, unspecific abdominal distribution via intraperitoneal administration, and mainly digestive distribution after intranasal administration, with some minor brain uptake (Fig. 6C). 


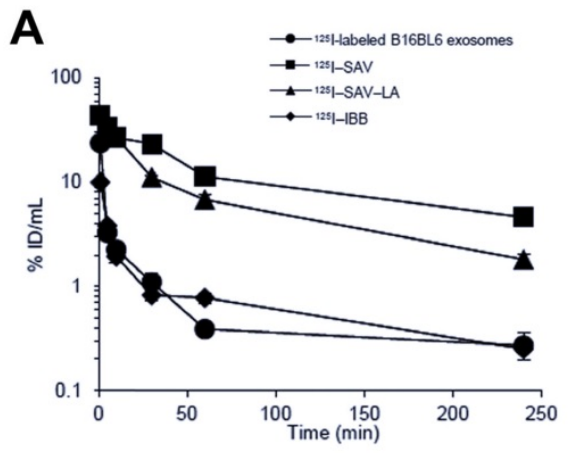

B

C

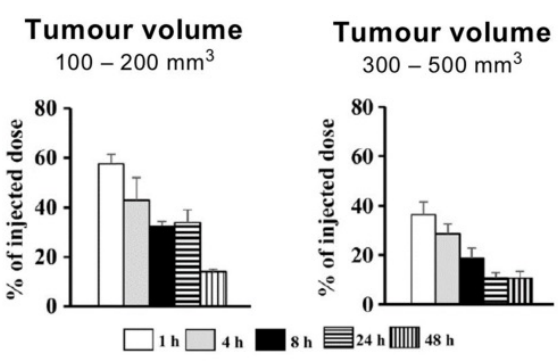

D

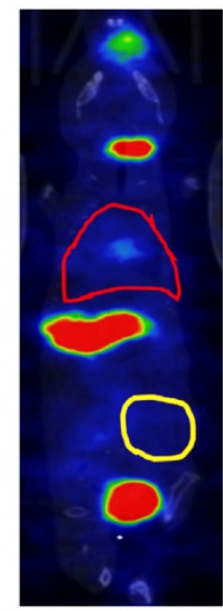

free $\mid-131$

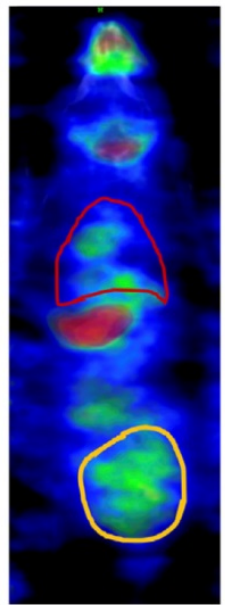

tumor exo

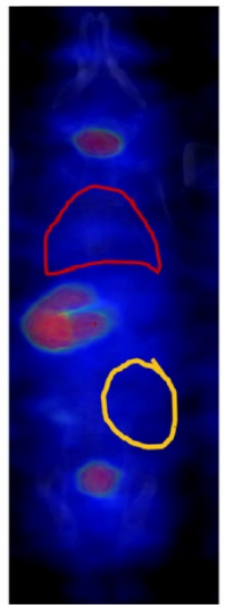

HEK293 exo

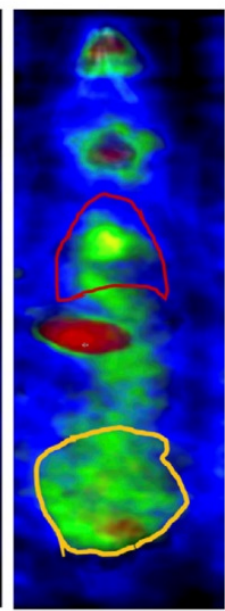

EPC exo

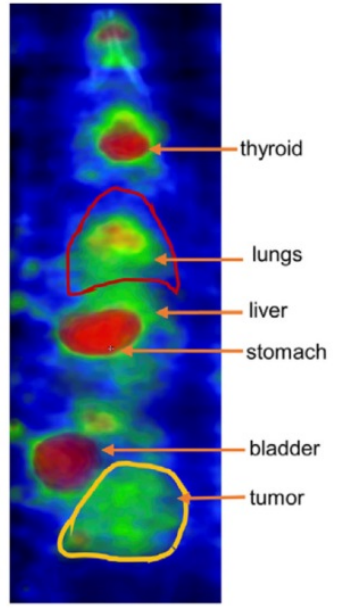

MDSC exo

Figure 5. (A) Blood clearance profile of ${ }^{125} \mid$-labelled B16-BL6 sEVs, [125|]I-SAV (streptavidin construct), [125|]I-SAV-LA (streptavidin-lactadherin fusion protein), and [125|]I-|BB (biotin conjugated radiotracer) in healthy mice after iv. injection; data presented as mean \pm standard error of means (SEM) of $n=4$. (B) Ex vivo biodistribution of 125 -labelled B16-BL6 sEVs and [125|]l-IBB over $4 \mathrm{~h}$ post iv. injection; data presented as mean \pm SEM of $n=4$. Figure taken with permission from Morishita et al. [78] (C) Retention of intratumorally injected 125 -labelled B16-BL6 sEVs in tumor tissues of a xenograft mouse model with tumour volume of $100-200$ or 300-500 mm3; data presented as mean \pm SEM of $n=4$. Figure adapted with permission from Matsumoto et al. [79] (D) In vivo biodistribution of I3II-labelled EVs (exo) isolated from 4T1 (mouse breast tumour) cells, HEK-293 (human embryonic kidney-293) cells, endothelial progenitor cells (EPC) and myeloid derived suppressor cells (MDSC) compared to free $131 /$ biodistribution in tumour bearing mice. Figure adapted with permission from Rashid et al. [80].

Despite being the most widely used radionuclide, the short half-life of ${ }^{99 m}$ Tc only allows imaging for up to $24 \mathrm{~h}$ post administration, which is not suitable for long term in vivo tracking of EVs. This can be resolved by using ${ }^{111}$ In $\left(t_{1 / 2}=2.8 \mathrm{~d}\right)$, another clinically available gamma-emitting radionuclide that is the second most commonly used for EV imaging after ${ }^{99 \mathrm{~m} T c}$ (Fig. 3). Smyth et al. used [111In]In-oxinate (intraluminal labelling) to radiolabel sEVs derived from PC3 $(140 \pm 59 \mathrm{~nm})$ and MCF7 $(130 \pm 57 \mathrm{~nm})$ cells, with RLY of $81 \%$ and $67 \%$, respectively [85]. These differences in RLY could be due to different affinities of the intraluminal composition of the two types of $\mathrm{sEVs}$ for the same radiometal. Blood clearance of the two sEVs was fast, and similar in tumour bearing mice as well as that of liposomes (Fig. 7A). High uptake in liver, spleen and kidneys, but low tumour uptake was reported in ex vivo data at $24 \mathrm{~h}$ for both healthy and tumour bearing mice (Fig. 7B). High kidney uptake could be a sign of "unchelated" ${ }^{111} \mathrm{In}$ release, but this was not evaluated. In a very interesting study, Rashid et al. also used intraluminal 
radiolabelling ([111In]In-oxinate) to track CD206-positive M2 macrophage-specific sEVs (106 \pm $14 \mathrm{~nm}$ ) in vivo using SPECT imaging [86]. The authors used [111In]In-oxinate to achieve very high RLY (98\%) and in vitro stabilities, as assessed by thin layer chromatography. In vivo SPECT-CT images using M2-targeted sEVs showed the expected liver and spleen uptake seen with other sEVs, but also interesting uptake in lymph nodes (Fig. 7C) - a finding that has also been observed with ${ }^{89} \mathrm{Zr}$-labelled PANC1 sEVs (vide infra) [93] - and lungs. In addition,
M2-targeted sEVs showed increased uptake in tumours, as well as significant kidney and bladder signal that the authors assign to excretion of radiolabelled sEVs. Although it would have been preferable to report image scales to allow image comparisons and quantify organ/tumour uptake using normalised standard units (ca. \% ID/mass, $\%$ ID/volume, SUV), instead of \%ID or counts/mass, these results show the potential of engineering sEVs for cell-specific targeting.

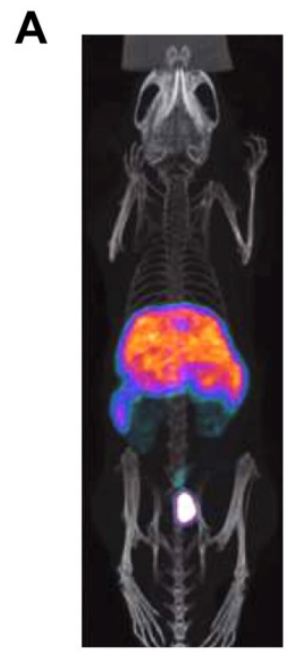

B

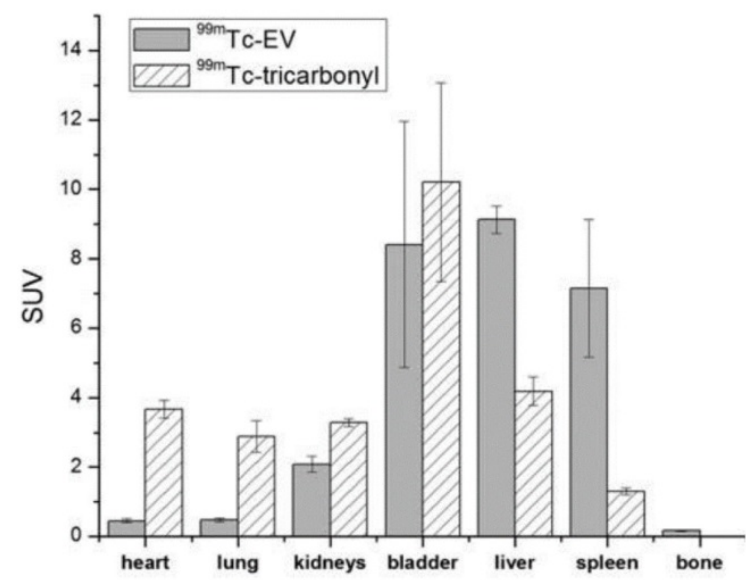

C
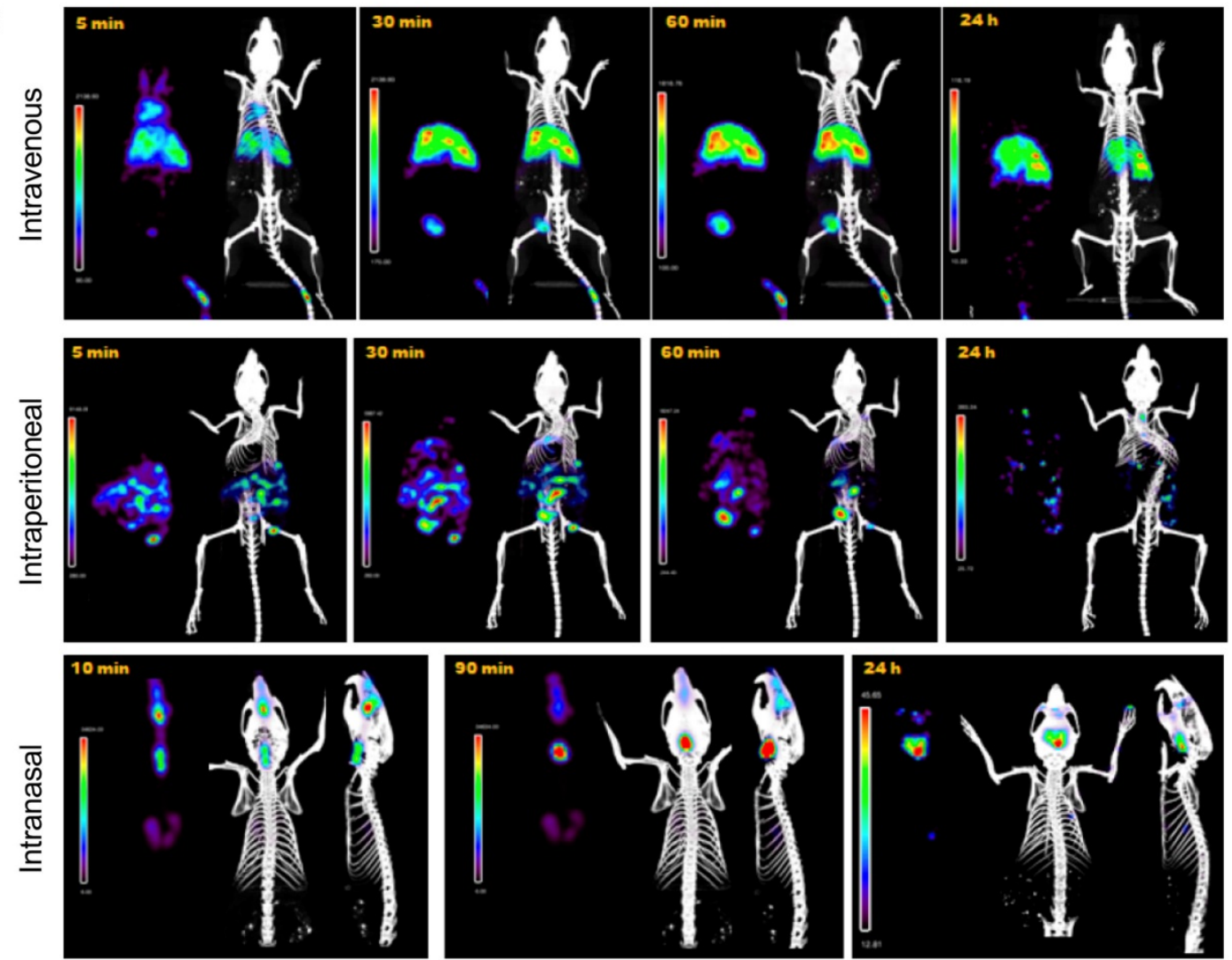

Figure 6. A) SPECT-CT images of [99mTc]T c-tricarbonyl labelled RBC derived sEVs. B) Ex vivo biodistribution of $99 \mathrm{mT}$ c-sEVs and [99mTc]Tc-tricarbonyl injected iv. in male BALB/c mice about $2 \mathrm{~h}$ post injection $(\mathrm{n}=3$ ). Figures taken with permission from Varga et al. [81] C) In vivo biodistribution of $99 \mathrm{mTc}$-labelled goat milk derived sEVs injected in healthy female BALB/c mice via three different administration routes over time as indicated on the images. Figure adapted with permission from González et al. [83]. 
A

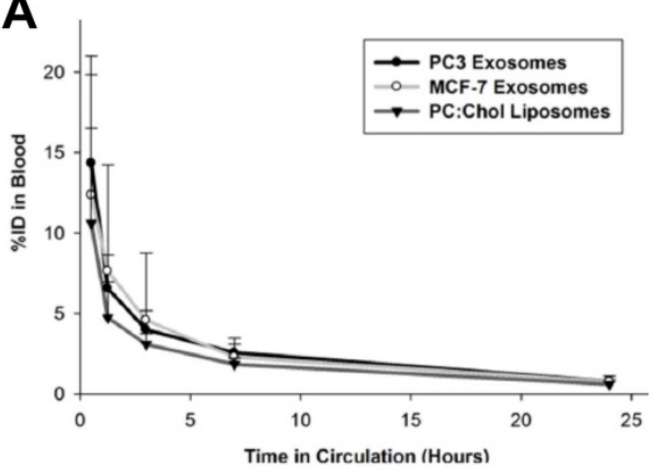

C

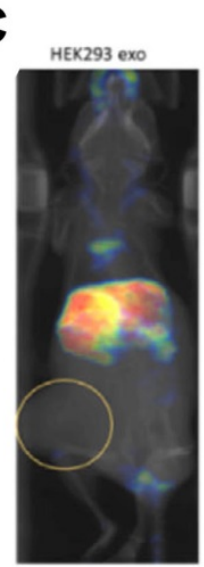

B

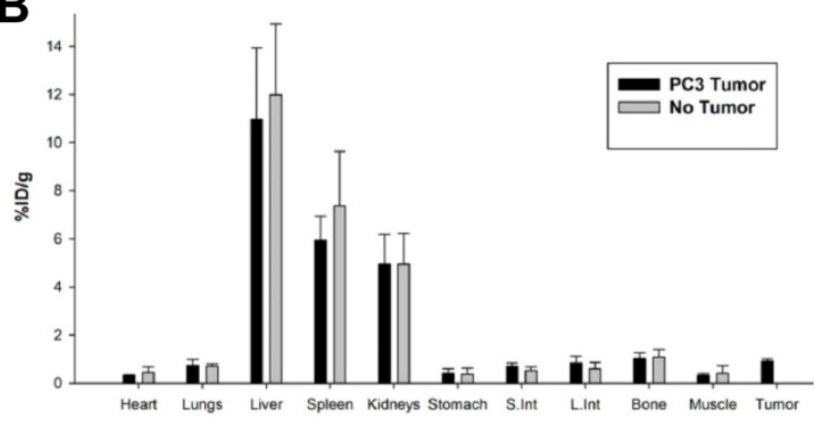

Figure 7. (A) Blood clearance of IIIIn-labelled PC3 (prostate cancer) and MCF-7 (breast cancer) sEVs, compared to liposomes. (B) Ex vivo biodistribution of IIIIn-labelled PC3 sEVs in PC3 tumor-bearing nude mice and non-tumor bearing mice $24 \mathrm{~h}$ post injection. Figures taken with permission from Smyth et al. [85] (C) In vivo biodistribution of iv. administered [ $\left.{ }^{\prime \prime \prime} I n\right]$ In-oxinate labelled CD206-positive M2 macrophage targeting sEVs (middle image) as well as control groups (left and right images) in tumour bearing BALB/c mice at $3 \mathrm{~h}$ post injection. Figure taken with permission from Rashid et al. [86] (D) Ex vivo biodistribution of iv. administered [11'In]ln-tropolone and [11'In]ln-DTPA labelled B16-F10 sEVs at $24 \mathrm{~h}$ post injection. Data presented as mean \pm SD of $\mathrm{n}=3$ and analysed by Student's t-test. Figure adapted with permission from Faruqu et al. [84].

Similar to [111In]In-oxinate, [111In]In-tropolone is another lipophilic radiotracer that allows intraluminal radiolabelling, but with higher inertness/stability compared to [111In]In-oxinate that makes it more resistant to transchelation, and hence potentially less effective for intraluminal radiolabelling. In fact, [111In]In-oxinate often results in higher cell RLYs when compared to [111In]In-tropolone [101]. Faruqu et al. used [111In]In-tropolone to achieve a RLY of $4.73 \pm$ $0.39 \%$ with B16-F10 sEVs $(132 \pm 6 \mathrm{~nm})$ [84]. In this study SEC using Sepharose ${ }^{\circledR}$ CL-2B resin was used to separate unbound radiotracer, a technique that potentially results in losses of $c a .50 \%$ of the vesicles. The authors also performed membrane radiolabelling of the same sEVs using [111In]In-DTPA (RLY = $19.2 \pm$ $4.5 \%)$. Overall, the pharmacokinetics and tissue biodistribution differed slightly depending on whether they were radiolabelled on the membrane or intraluminally (Fig. 7D). The relatively lower radiochemical stability of the [111In]In-tropolone labelled sEVs was evident in the in vivo biodistribution with lower liver/spleen retention. This study also demonstrated that there are no significant differences in SEV biodistribution between immunocompetent (C57BL/6) and immunocompromised (NOD SCID gamma (NSG)) mice, except minor differences in tumour uptake, that could be explained by the smaller population of tumour associated macrophages in NSG mice.

\section{Radiolabelling of EVs using PET radionuclides}

It is well established that intra-vesicular nucleic acids and proteins can be used as biomarkers for various diseases. Recently, it has been reported that the glycan profiles of EVs can also be used as cancer biomarkers [102]. Moreover, enrichment of specific glycoproteins, such as sialic acid [103], allow iv. injected EVs to be captured by CD169 positive macrophages in spleen and lymph nodes [104]. Royo et al. evaluated the effect of glycosylation on sEV biodistribution by radiolabelling them with ${ }^{124} \mathrm{I}\left(t_{1 / 2}=\right.$ $4.2 \mathrm{~d}$ ) [89]. Using iodination tubes (iodogen method), which allows oxidation of ${ }^{124} \mathrm{I}$ and thus radiolabels proteins on cell membranes [95], and a RLY of $>15 \%$, they have shown that modified glycosylation allows 
sEVs to accumulate and retain in the lungs after iv. injection; and to migrate through the lymph system after hock injection (injection in the joints), even though a large amount was retained in the injection site (Fig. 8A). The in vivo PET imaging data showed that sEVs start accumulating in the liver as early as 30 $\mathrm{s}$ after iv. injection, and joint administration results in expected lymphatic drainage. Although it is difficult to compare the in vivo images without appropriate scale bars; there were only minor differences (lung uptake) between glycosylated or non-glycosylated sEVs, with both showing increasing signal in bladder and thyroid over time, the latter being a sign of in vivo deiodination (Fig. 8A-B). Continuing with membrane labelling, Banerjee et al. used $\left[{ }^{64} \mathrm{Cu}\right] \mathrm{Cu}$-DOTAMaleimide $\left(t_{1 / 2}=12.7 \mathrm{~h}\right)$ to radiolabel sEVs $(\sim 110 \mathrm{~nm})$ [90]. DOTA was attached on the sEVs' surface using endogenous membrane thiol groups. This allowed $c a$. $20 \%$ RLY of umbilical cord blood cell sEVs. Interestingly, in vivo imaging showed brain uptake 20 - 60 min post injection (Fig. 8C). A considerable amount of sEV uptake was seen in the bladder, which increased over time as the liver signal decreased (Fig. 8D). Interestingly adding a further SEC purification following ultracentrifugation increased $\mathrm{sEV}$ accumulation in liver and spleen but decreased in urine/bladder (discussed in the "Challenges" section below).

Facilitated by the specific glycan profile, the rapid uptake of EVs by liver and spleen, results in very short blood circulation time when injected iv. $[105$, 106]. PEGylation is commonly used in nanomedicine to overcome such issues [107]. Likewise, PEGylated EVs have been shown to improve circulation, reduce liver sequestration and improve tumour uptake [108]. Shi et al. was the first group to study in vivo biodistribution of PEGylated EVs, using $\left.{ }^{64} \mathrm{Cu}\right] \mathrm{Cu}-\mathrm{NOTA}$ [91]. Using the same (or less) amount of sEVs ( $106 \mathrm{~nm})$ as Banerjee and his group, they achieved considerably higher RLY (91.2 \pm $0.2 \%$ vs. $\sim 20 \%$ ). This could be the result of the well-known high radiochemical stability of $\left[{ }^{64} \mathrm{Cu}\right] \mathrm{Cu}-\mathrm{NOTA}$ complexes $[109,110]$. PEGylation had the desired effect of increased sEV circulation, decreased liver uptake and increased tumour uptake, particularly after $24 \mathrm{~h}$ (Fig. 8E-F). Uptake in the lymph nodes (ca. 2\% ID/g) for both PEGylated and non-PEGylated was also identified from the ex vivo biodistribution data, but not discussed further (Fig. 8G). In another report involving surface radiolabelling with PET radionuclide, Jung et al. isolated sEVs from $4 \mathrm{~T} 1$ breast cancer cells $(\sim 100 \mathrm{~nm})$ and radiolabelled them with ${ }^{64} \mathrm{Cu}$ and ${ }^{68} \mathrm{Ga}$, via NOTA-isothiocyanate conjugation to sEV surface amine groups (from surface proteins) and also labelled with Cy7 to allow optical imaging [92]. RLY with ${ }^{64} \mathrm{Cu}$ was $>98 \%$, which is comparable to what was reported by Shi et al. RLY and serum stability of $>$ $95 \%$ by TLC were only reported for ${ }^{64} \mathrm{Cu}$-labelled sEVs. Healthy mice were injected iv. or subcutaneously. As expected from this administration route, lymph node uptake was observed after subcutaneous injection, and the PET signal correlates well the fluorescent signal. High uptake in the lungs, liver and spleen was observed for iv. injected sEVs. Furthermore, presence of CD63 and Cy7 positive sEVs in liver, spleen and lymph nodes were confirmed by histological analysis.

To date there is one report on intraluminal radiolabelling of sEVs using PET radionuclides [93]. Khan et al. exploited the proven cell and liposome radiolabelling capabilities of [ $\left.{ }^{89} \mathrm{Zr}\right] \mathrm{Zr}$-oxinate [77, 111, 112], to radiolabel three different cancer cell-derived sEVs with RLYs as high as $c a .20 \%$ for $1 \times 10^{11}$ PANC1 sEVs (97 $\pm 4 \mathrm{~nm}$ ) (Fig. 9A-B), and ca. 76\% radiochemical stability in PBS over $26 \mathrm{~h}$. An alternative in vitro stability was demonstrated in a cell-uptake study in the presence of serum proteins, showing significant differences between cell lines over $4 \mathrm{~h}$ in the uptake of ${ }^{89} \mathrm{Zr}-\mathrm{PANC} 1 \mathrm{sEVs}$ vs. control groups. In vivo PET imaging in healthy mice showed the expected biodistribution of sEVs with uptake in liver and spleen, and interestingly several lymph nodes and brain, particularly at early timepoints (Fig. 9C-D). Furthermore, significant differences were observed in the uptake of ${ }^{89} \mathrm{Zr}-\mathrm{PANC} 1 \mathrm{sEVs}$ and heat-damaged ${ }^{89} \mathrm{Zr}-\mathrm{PANC1}$ sEVs. The latter group was aimed at denaturing the radiolabelled PANC1 sEVs to study the effect in the biodistribution. In particular, significant lower spleen uptake was observed at all time-points, leading to the suggestion that spleen/bone and liver/bone ratios may be used as in vivo markers of stability of sEVs radiolabelled using [ ${ }^{89} \mathrm{Zr}$ ]Zr-oxinate (Fig. 9E).

\section{Radiolabelling of exosome mimetic vesicles (EMVs)}

Variability in EV production, isolation and radiolabelling properties can be overcome by using exosome mimetic vesicles (EMVs). EMVs are produced mainly by serial extrusion of cell membrane, and so far used for radiolabelling [113], but can also be produced by other methods [68]. The main advantage of EMVs is that they can be mass produced in larger concentration than EVs. They are comparable to EVs in size, protein and lipid composition, biomarker expression, and retain tissue targeting properties. 
A
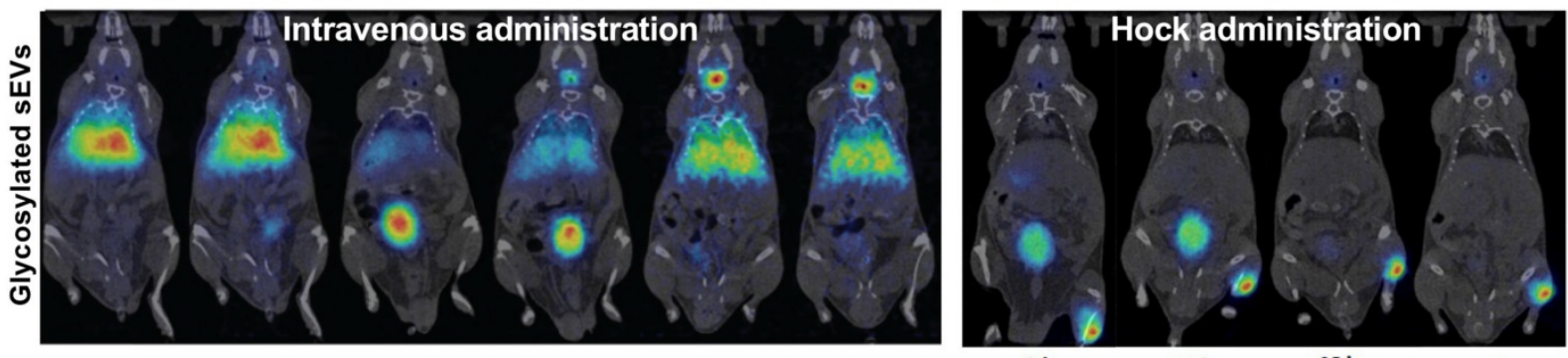

$20 \mathrm{~min} \quad 40 \mathrm{~min}$

$7 \mathrm{~h}$

$24 \mathrm{~h}$

$48 \mathrm{~h}$

$72 \mathrm{~h}$

$3 \mathbf{h}$

$24 \mathrm{~h}$

$72 \mathrm{~h}$
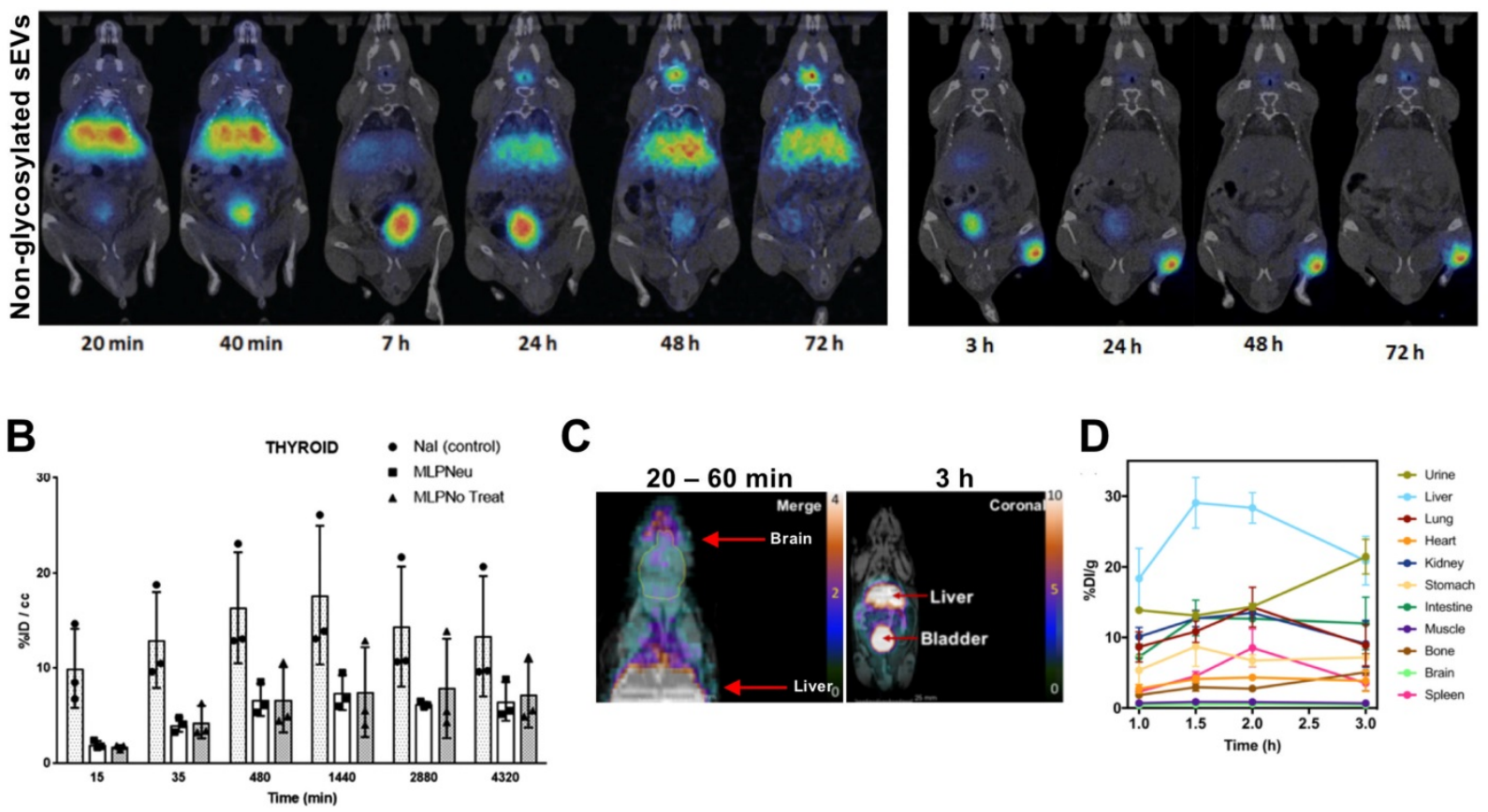

C

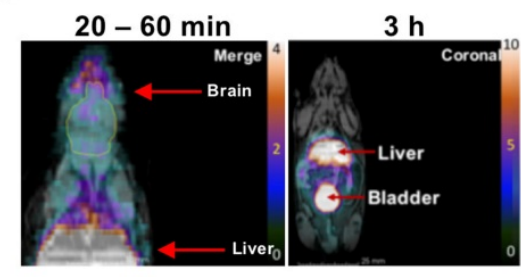

D

E

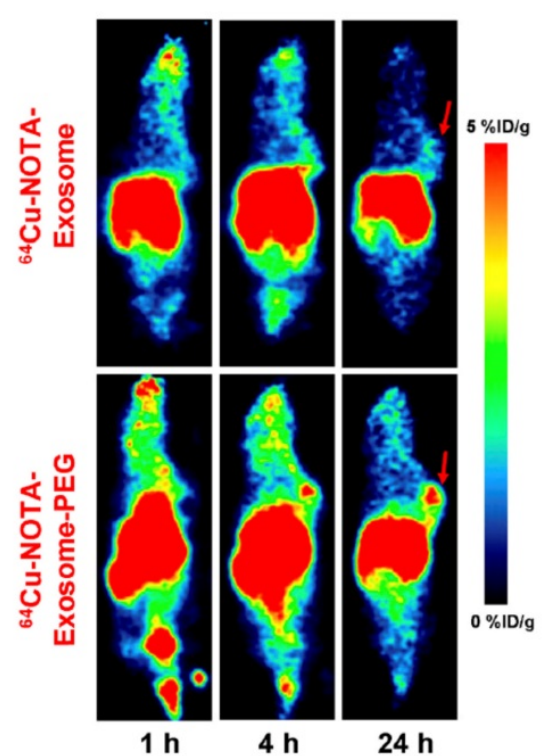

F

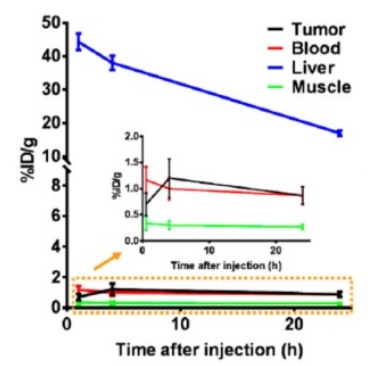

G

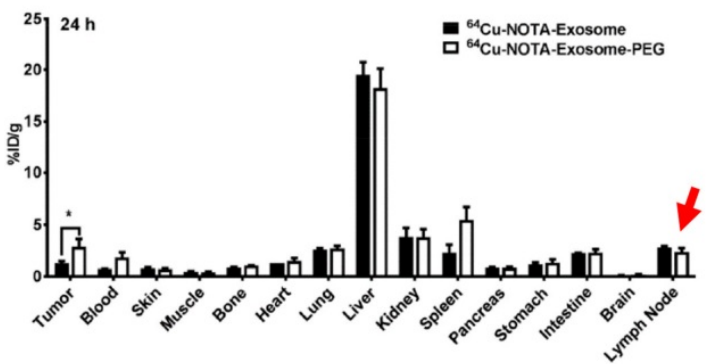

Figure 8. (A) In vivo biodistribution of 124 -labelled MLP29 sEVs following iv. and hock administration. (B) Ex vivo biodistribution of $124 \mid$-labelled MLP29 sEVs in the thyroid over time for $\mathrm{Nal}$ (control), glycosylated sEVs (MLPNeu) and non-glycosylated sEVs (MLPNo Treat) following iv. administration, data given as mean \pm standard deviation (SD) of $n=$ 3. Figures adapted with permission from Royo et al. [89] (C) PET-MR images of iv. administered [ $\left.{ }^{64} \mathrm{Cu}\right] \mathrm{Cu}-\mathrm{DTPA}$ labelled sEVs $20-60 \mathrm{~min}$ and $3 \mathrm{~h}$ post injection. (D) Ex vivo biodistribution of [ ${ }^{64} \mathrm{Cu}$ ]Cu-DTPA labelled sEVs at $1,1.5$ and $2 \mathrm{~h}$ post injection; data represented as mean \pm SEM of $n=3-4$. Figures adapted with permission from Banerjee et al. [90] (E) In vivo PET images, and (F) time activity curves of iv. administered [ ${ }^{64} \mathrm{Cu}$ ]Cu-NOTA labelled 4T1 sEVs comparing the biodistribution of PEGylated and non-PEGylated $s E V s$ in $4 T 1$ tumour bearing female BALB/c mice over time. (G) Ex vivo biodistribution of ${ }^{64} \mathrm{Cu}-4 \mathrm{~T} 1 \mathrm{sEV}$, PEGylated or non-PEGylated, in $4 T 1$ tumour bearing female BALB/c mice $24 \mathrm{~h}$ post injection. Red arrow highlights lymph node uptake. Data expressed as mean \pm SD of $\mathrm{n}=3$. Figures taken with permission from Shi et al. [91]. 
A

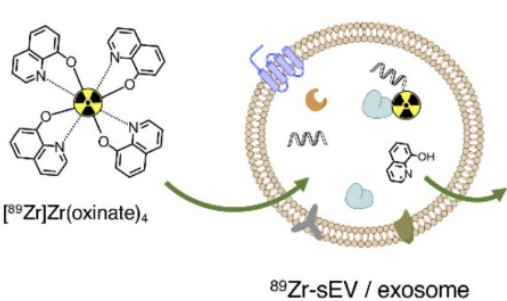

C

89Zr-sEV / exosome

B
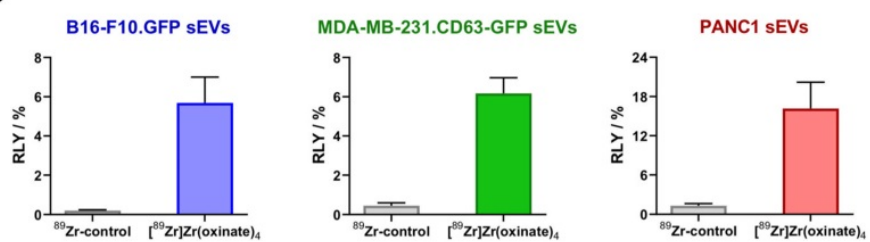

D

(iii) $89 \mathrm{Zr}$-control

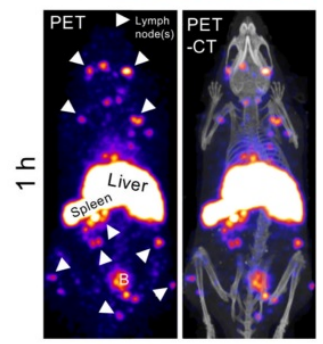

(ii) Heat damaged
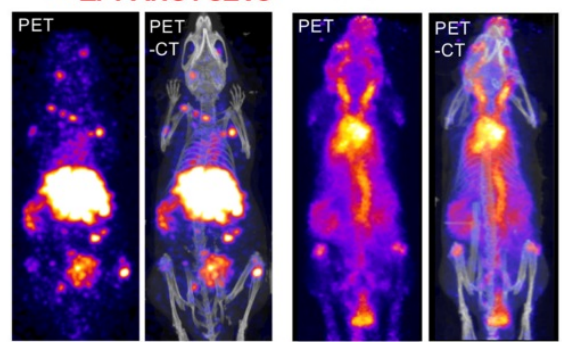

89Zr-PANC1 sEVs $1.5 \mathrm{~h}$ p.i.
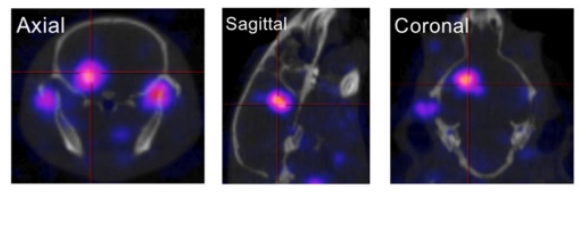

E

Ex vivo biodistribution at $24 \mathrm{~h}$ p.i.

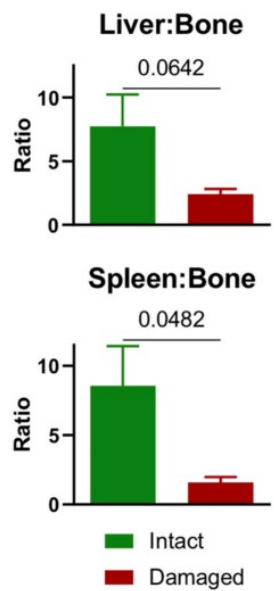

Figure 9. (A) Schematic representation of intraluminal radiolabelling of sEVs using $\left.{ }^{89} \mathrm{Zr}\right] \mathrm{Zr}$-oxinate. (B) Radiolabelling yield (RLY) of $1 \times 1010 \mathrm{~B} 16-\mathrm{F} 10 . \mathrm{GFP}$ sEVs, $1 \times 1010$ MDA-MB-231.CD63-GFP sEVs and $1 \times 1011$ PANC1 sEVs; data given as mean \pm SD of $n=3$. (C) In vivo PET-CT images of C57BL/6 mice injected iv. with $89 \mathrm{Zr}-\mathrm{PANC1} \mathrm{sEV}$, heat-damaged ${ }^{89} \mathrm{Zr}$-PANCl sEVs and ${ }^{89} \mathrm{Zr}$-control, $1 \mathrm{~h}$ post injection; $\mathrm{B}=$ bladder, $\dagger=\mathrm{PET}$ image scale for ${ }^{89} \mathrm{Zr}$-control is 10 times that of the other images; adjusted for image clarity. (D) Image slices of a mouse injected with intact $89 \mathrm{Zr}$-PANCl sEVs showing uptake in brain; image scale is the same as in $\mathbf{C}$. (E) Ex vivo biodistribution of "intact" ( $\mathrm{n}=3$ ) and "heat-damaged" $(n=2){ }^{89} \mathrm{Zr}$-PANCl sEVs. Ratio of liver:bone uptake $(n=3)$ and spleen:bone uptake $(n=2)$ is shown on the right; data given as mean \pm SD of the $n$ values and analysed by Student's t-test. Figures taken from Khan et al. [93].

Using mouse macrophage derived EMVs $(218 \pm$ $8 \mathrm{~nm})$, Hwang et al. managed to achieve RLY of $>93 \%$ with [99mTc]Tc-HMPAO $\left(t_{1 / 2}=6 \mathrm{~h}\right)$ [87]. They utilised the endogenous intra-vesicular thiol groups to convert the lipophilic [ ${ }^{99 \mathrm{mTc}}$ ]Tc-HMPAO complex into a hydrophilic one, thus trapping the radionuclide in the intraluminal space, unlike the surface thiol groups used previously by Banerjee et al. In vivo biodistribution of two different types of ${ }^{99 \mathrm{mTc}} \mathrm{T}$-labelled EMVs were compared with mouse macrophage derived sEVs, and although comparisons are difficult from the images reported, it seems that significant release of the [99mTc]-HMPAO complex and/or $\left[{ }^{99} \mathrm{TcO}_{4}\right]^{-}$is evident (Fig. 10A). Later on, Gangadaran et al. also studied biodistribution of $99 \mathrm{mTc}$-labelled RBC derived EMVs $(201 \pm 16 \mathrm{~nm})$. Their radiolabelling hypothesis is based on the RBC radiolabelling protocol, where ${ }^{99} \mathrm{mc}^{4+}$ reduced by $\mathrm{SnCl}_{2}$ binds to intercellular haemoglobin [114]. In this case, ${ }^{99 \mathrm{~m} T c}$ bound to haemoglobin inside the EMVs (Fig. 10B). With $\sim 100 \%$ RLY after 20 min incubation, no further purification was used. Healthy C57BL/ 6 mice were injected iv. with 99mTc-labelled EMVs and gamma camera imaging was performed, as opposed to SPECT. Nonetheless, no significant difference was observed between $1 \mathrm{~h}$ and $3 \mathrm{~h}$ post injection for the EMVs, unlike for free ${ }^{99 \mathrm{~m} T c}$. Once again, significant signal from the liver/spleen and bladder was observed with the radiolabelled EMVs. (Fig. 10C-D). Subsequently, due to the efficiency and stability of radiolabelling, the group used the $99 \mathrm{mTc}$-labelled EMVs to successfully radiolabel and track white blood cells in vivo [115]. 
A
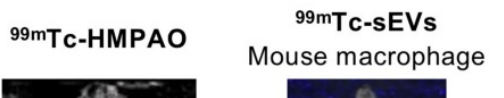

99mTc-EMVs

99mTc-EMVs
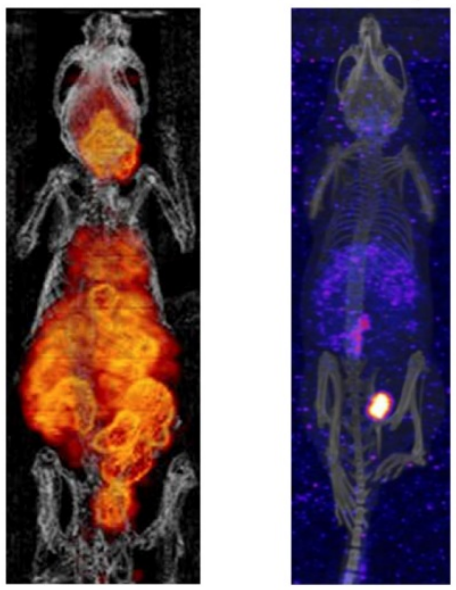

Mouse macrophage

Human neural stem cell
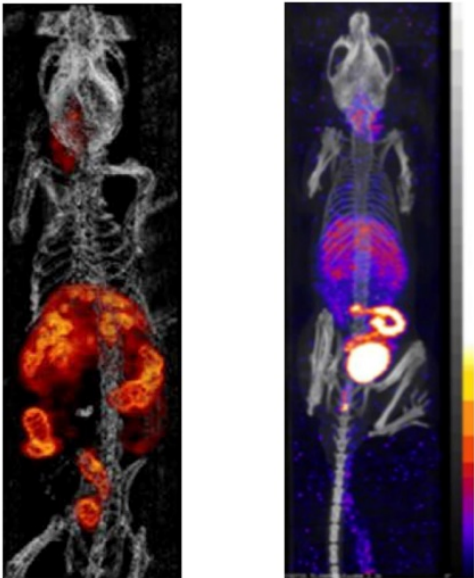

B
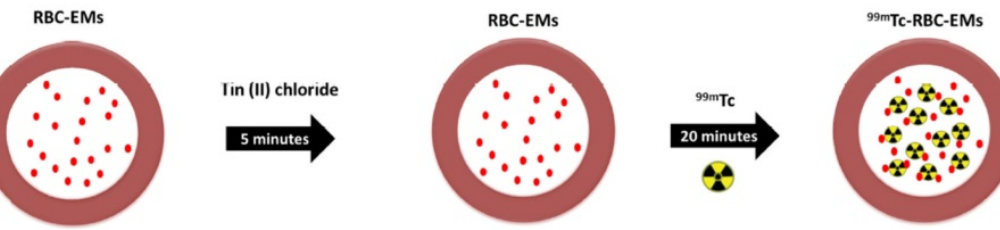

C

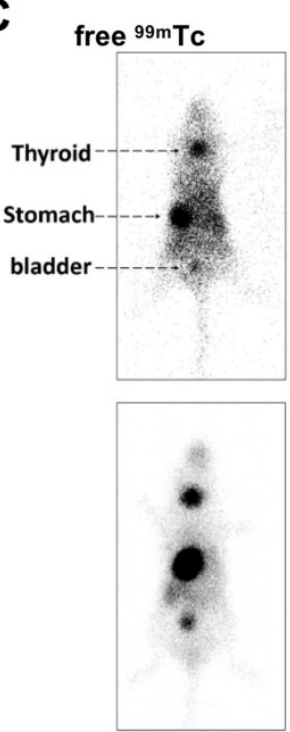

Iin (II) chloride

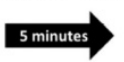

D

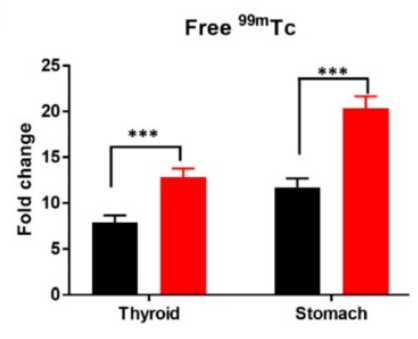

${ }^{99 \mathrm{~m}}$ TC-RBC-EMs

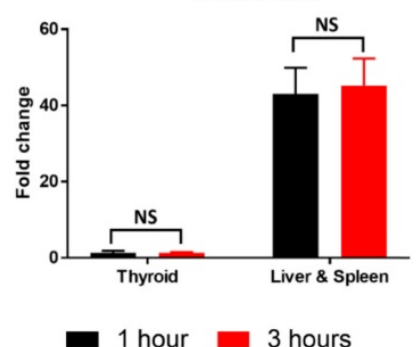

Figure 10. (A) In vivo SPECT-CT images of 99mTc-labelled EMVs derived from mouse macrophages and human neural stem cells, compared with $99 \mathrm{mT}$-labelled sEVs derived from mouse macrophages and free [99mTc]Tc-HMPAO $3 \mathrm{~h}$ post injection. Figures taken with permission from Hwang et al. [87] (B) Schematic representation of the protocol for radiolabelling red blood cell-derived EMVs with $99 \mathrm{mTc}$. (C) In vivo gamma camera images of iv. administered free $99 \mathrm{mTc}$ and $99 \mathrm{mTc}$-EMVs in male C57BL/6 mice $1 \mathrm{~h}$ and $3 \mathrm{~h}$ post injection. (D) Quantification of free $99 \mathrm{mTc}$ and $99 \mathrm{mTc}-E M V s$ injected in organs of interest $1 \mathrm{~h}$ and $3 \mathrm{~h}$ post injection; data presented as mean \pm SD of $\mathrm{n}=4$ and analysed by Student's t-test. Figures adapted with permission from Gangadaran et al. [88].

\section{Challenges in the radiolabelling and in vivo SPECT/PET imaging of EVs}

One of the biggest challenges in the in vivo imaging of EVs is their instability. A study by Clayton et al. demonstrated abundant presence of CD55 and CD59 on EVs, that could lend themselves longer survivability in vivo [72]. However, according to the EV radiolabelling studies discussed above, as well as studies using non-radiolabelled EVs [104, 116], blood half-life of iv. administered EVs was found to be as short as $<2 \mathrm{~min}$. However, presence of EVs could be detected in the reticuloendothelial system (RES) organs, particularly in liver and spleen, long after clearance from blood.

After liver and spleen, the highest accumulation of imaging signals is observed in the bladder; only possible if the EVs are able to pass through the glomerular filtration in the kidneys. Using quantum dots, Choi et al. demonstrated that, nanoparticles $>8$ 
nm are not typically cleared by the kidneys [117]. This study suggested that the renal filtration size threshold maybe comparable to small proteins and was further demonstrated by antibody clearance data. Thus, renal clearance of intact antibodies is considered insignificant because the size of a typical antibody (e.g. $\sim 150 \mathrm{kDa}$ for $\mathrm{IgG})$ is much larger than the glomerular filtration threshold ( $\sim 55 \mathrm{kDa})$ (Fig. 11A) [118]. Antibody fragments, on the other hand, are much smaller (for example, the size of the Fab fragment of $\operatorname{IgG}$ is $\sim 50 \mathrm{kDa}$ ) and are cleared by kidneys [119]. Hence it seems to us that the kidney/bladder uptake observed in many SPECT/PET imaging studies with radiolabelled EVs is likely to be the result from fast disintegration of EVs in blood/serum. The radioactive hot-spots seen in bladder in the SPECT and PET images are either from the radiotracer itself (that taking into account that bladder uptake is often observed, regardless of radiolabelling method, makes this possibility improbable in many cases) or from EV components that are attached to the radiotracer. Interestingly, the supplementary data reported by Banerjee et al. [90], showing that EV purification by combining ultracentrifugation with SEC leads to lower accumulation in urine, seems to support this hypothesis (Fig. 11B). Previous studies have shown that $\mathrm{sEVs}$ isolated by ultracentrifugation can lead to co-precipitation of serum proteins (such as albumin) [120], with lower purity as determined by the particle-to-protein ratio, compared to SEC [121] despite co-isolation of lipoproteins using this method [122]. Wei et al. have shown that by combining these two methods, it is possible to improve elimination of contaminating proteins, lipoproteins and to improve particle-to-protein ratio [123]. Nevertheless, further evidence is needed to determine if this is the case for kidney/bladder/urine uptake of EVs.

Another of the main issues with many EV radiolabelling studies reviewed above is the use of instant thin layer chromatography (iTLC) to measure radiochemical stability in serum. When using appropriate stationary and mobile phases, for example Whatman No 1 paper as the stationary phase and ethyl acetate or EDTA as the mobile phase for $\left[{ }^{89} \mathrm{Zr}\right] \mathrm{Zr}$-oxinate, the lipophilic unbound radiotracer or leaked ${ }^{89} \mathrm{Zr}^{4+}$ ions migrates to the solvent front $\left(R_{\mathrm{f}}=\right.$ 1) respectively, whereas radioactivity bound to the EVs stays at the origin $\left(R_{f}=0\right)$ because EVs are not soluble and precipitate in this solvent system. This technique is appropriate when assessing radiochemical stability in aqueous buffers such as PBS. On the other hand, in serum, any trans-chelation of the radiotracer by the serum proteins would also be detected at the origin because proteins will also precipitate in the presence of organic solvents, even at low concentrations; making it difficult to distinguish whether the radioactivity is bound to EVs or serum proteins.

\section{Conclusions and perspectives}

The field of EV research and in particular the applications of EVs as nanomedicinal tools has sparked a recent remarkable interest from many researchers. From all the research carried out to this effect, it is clear that using imaging techniques, whether optical or radionuclide-based, is certainly beneficial in elucidating EV biology and behaviour, and should be integrated into this research early in the process to facilitate their development as potential therapies/diagnostic tools.
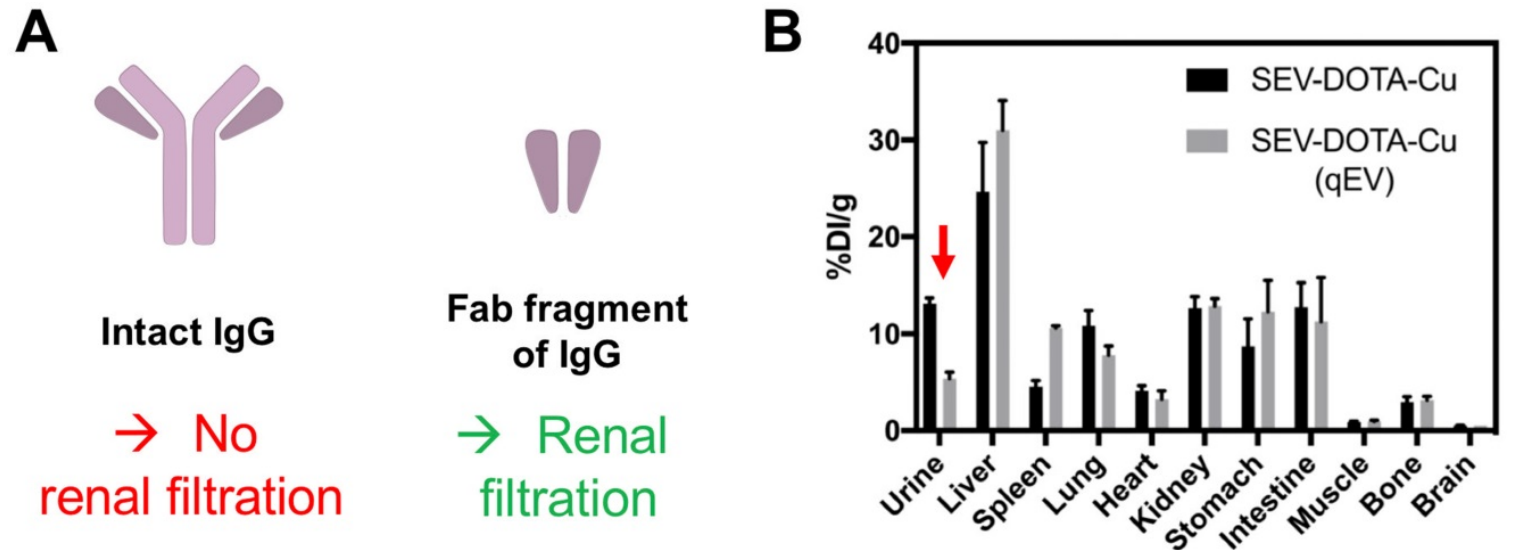

Figure 11. (A) Schematic structures of an intact $\lg \mathrm{G}$ antibody $(\sim 150 \mathrm{kDa})$ and its Fab fragment $(\sim 50 \mathrm{kDa})$. (B) Ex vivo biodistribution of ${ }^{64} \mathrm{Cu}-\mathrm{labelled}$ sEVs isolated by ultracentrifugation only vs. ultracentrifugation combined with SEC (using a qEV column). Red arrow indicating the difference in urine signal. Figure taken with permission from Banerjee et al. [90]. 
In this review we focussed on EV research to date that includes radionuclide-based imaging, and in particular on the radiolabelling methods used and findings from SPECT/PET imaging. In these studies, two main categories of radiolabelling methods were used - intraluminal and membrane-based. Our review illustrates the importance of choosing the most appropriate radiolabelling method suitable for the downstream application, as each have their advantages and disadvantages. Of all these radiolabelling methods, two main ones stand out in terms of efficiency and stability: (i) direct conjugation of a bifunctional radionuclide-complex (e.g. NOTA-maleimide) onto surface components and (ii) the use of ionophores such as oxine for intraluminal labelling. Ideally, studies that compare these two methods such as that from Faruqu et al. should be performed prior to any PET/SPECT EV study to identify the best suited method for each EV and application [84].

It is important to emphasise that when radiolabelling EVs via the surface/membrane, we will be tracking these membrane components over time, whether they are still a part of the EV or not. Likewise, when we radiolabel intraluminally we will only be tracking the EVs as long as their contents have not been released. For this reason, regardless of the radiolabelling method, it is important to have a clear understanding of the biodistribution/pharmacokinetics of the radionuclide and/or the radio-complex used for EV radiolabelling. We think this is a crucial component of any EV PET/SPECT imaging experiment as many radionuclides/radio-complexes share biodistribution and excretion pathways with nanoparticulates (e.g. liver, lymph nodes, tumours) [69]. Ideally, the radiolabelling method of choice should not affect the natural properties of EVs (e.g. hydrodynamic diameter, membrane protein structure and composition, colloidal stability), and result in physicochemically and radiochemically stable products. In this regard, we believe there is a significant risk when using direct attachment of radionuclides or radionuclide-based bifunctional chelators onto the surface of EVs, as these are likely to affect the structure of important surface proteins/molecules that the EVs use for their function.

One of the main findings of this review is the high number of studies that show accumulation of imaging signal/EVs in the bladder, making it unlikely that this is simply due to impurities from the radiolabelling process. As we discussed in the previous section, we believe that this may be linked to small EV fragments as a result of fast EV decomposition. Further studies are required to test this hypothesis, and we believe that radionuclide imaging will play an important role in evaluating this.

Finally, it is commonly accepted in the EV field that even a carefully isolated pure microvesicle or exosome population can be highly heterogeneous, and contains subgroups of vesicles with distinct properties [26, 124, 125]. This highlights the benefits of integrating in vivo EV research with radionuclidebased imaging (that benefits from high sensitivity and quantification properties) to allow us improving our understanding of the basic EV biology of each of these subgroups and their interaction with other cells/tissues. In addition, being a clinically available imaging modality offers the possibility to develop/optimise the methodology for potential future clinical studies.

\section{Acknowledgements}

Azalea Khan is supported by the UK Medical Research Council (MRC) and King's College London MRC Doctoral Training Partnership in Biomedical Sciences [MR/N013700/1]. The authors also acknowledge funding by EPSRC programme grants $\mathrm{EP} / \mathrm{S} 032789 / 1$ and EP/R045046/1, the Wellcome/ EPSRC Centre for Medical Engineering at KCL [WT/203148/Z/16/Z], the KCL and UCL Comprehensive Cancer Imaging Centre funded by CRUK and EPSRC in association with the MRC and DoH (England), and the National Institute for Health Research (NIHR) Biomedical Research Centre based at Guy's and St Thomas' NHS Foundation Trust and KCL [IS-BRC-1215-20006]. The views expressed are those of the authors and not necessarily those of the NHS, the NIHR or the Department of Health.

\section{Supplementary Material}

Supplementary materials. http://www.ntno.org/v05p0256s1.pdf

\section{Competing Interests}

The authors have declared that no competing interest exists.

\section{References}

1. Colombo M, Raposo G, Théry C. Biogenesis, Secretion, and Intercellular Interactions of Exosomes and Other Extracellular Vesicles. Annu Rev Cell Dev Biol. 2014; 30: 255-89.

2. Yáñez-Mó M, Siljander PRM, Andreu Z, Bedina Zavec A, Borràs FE, Buzas EI, et al. Biological properties of extracellular vesicles and their physiological functions. J Extracell Vesicles. 2015; 4: 27066.

3. Théry C, Ostrowski M, Segura E. Membrane vesicles as conveyors of immune responses. Nat Rev Immunol. 2009; 9: 581-93.

4. Maas SLN, Breakefield XO, Weaver AM. Extracellular Vesicles: Unique Intercellular Delivery Vehicles. Trends Cell Biol. 2017; 27: 172-88.

5. Raposo G, Nijman HW, Stoorvogel W, Liejendekker R, Harding CV, Melief CJ, et al. B lymphocytes secrete antigen-presenting vesicles. J Exp Med. 1996; 183: 1161-72.

6. Valadi H, Ekstrom K, Bossios A, Sjostrand M, Lee JJ, Lotvall JO. Exosome-mediated transfer of mRNAs and microRNAs is a novel mechanism of genetic exchange between cells. Nat Cell Biol. 2007; 9: 654-9. 
7. Raposo G, Stoorvogel W. Extracellular vesicles: exosomes, microvesicles, and friends. J Cell Biol. 2013; 200: 373-83.

8. Suetsugu A, Honma K, Saji S, Moriwaki H, Ochiya T, Hoffman RM. Imaging exosome transfer from breast cancer cells to stroma at metastatic sites in orthotopic nude-mouse models. Adv Drug Deliv Rev. 2013; 65: 383-90.

9. Webber J, Yeung V, Clayton A. Extracellular vesicles as modulators of the cancer microenvironment. Semin Cell Dev Biol. 2015; 40: 27-34.

10. Zhao H, Achreja A, Iessi E, Logozzi M, Mizzoni D, Di Raimo R, et al. The key role of extracellular vesicles in the metastatic process. Biochim Biophys Acta Rev Cancer. 2018; 1869: 64-77.

11. Milman N, Ginini L, Gil Z. Exosomes and their role in tumorigenesis and anticancer drug resistance. Drug Resist Updat. 2019; 45: 1-12.

12. Xiao T, Zhang $W$, Jiao B, Pan CZ, Liu $X$, Shen $L$. The role of exosomes in the pathogenesis of Alzheimer' disease. Transl Neurodegener. 2017; 6.

13. Saeedi S, Israel S, Nagy C, Turecki G. The emerging role of exosomes in mental disorders. Transl Psychiatry. 2019; 9: 122.

14. Xiao Y, Zheng L, Zou X, Wang J, Zhong J, Zhong T. Extracellular vesicles in type 2 diabetes mellitus: key roles in pathogenesis, complications, and therapy. J Extracell Vesicles. 2019; 8: 1625677.

15. Buzas EI, György B, Nagy G, Falus A, Gay S. Emerging role of extracellular vesicles in inflammatory diseases. Nature Reviews Rheumatology. 2014; 10: 356-64

16. Oggero S, Austin-Williams S, Norling LV. The Contrasting Role of Extracellular Vesicles in Vascular Inflammation and Tissue Repair. Front Pharmacol. 2019; 10

17. Console L, Scalise M, Indiveri C. Exosomes in inflammation and role as biomarkers. Clin Chim Acta. 2019; 488: 165-71.

18. Keener A. How extracellular vesicles can enhance drug delivery. Nature. 2020; 582: S14-S5

19. Johnstone RM, Adam M, Hammond JR, Orr L, Turbide C. Vesicle formation during reticulocyte maturation. Association of plasma membrane activities with released vesicles (exosomes). J Biol Chem. 1987; 262: 9412-20.

20. Thery C, Zitvogel L, Amigorena S. Exosomes: composition, biogenesis and function. Nat Rev Immunol. 2002; 2: 569-79.

21. Heijnen HF, Schiel AE, Fijnheer R, Geuze HJ, Sixma JJ. Activated platelets release two types of membrane vesicles: microvesicles by surface shedding and exosomes derived from exocytosis of multivesicular bodies and alpha-granules. Blood. 1999; 94: 3791-9.

22. Muralidharan-Chari V, Clancy JW, Sedgwick A, D'Souza-Schorey C. Microvesicles: mediators of extracellular communication during cancer progression. J Cell Sci. 2010; 123: 1603-11.

23. Caruso S, Poon IKH. Apoptotic Cell-Derived Extracellular Vesicles: More Than Just Debris. Front Immunol. 2018; 9: 1486.

24. Li P, Kaslan M, Lee SH, Yao J, Gao Z. Progress in Exosome Isolation Techniques. Theranostics. 2017; 7: 789-804.

25. Kowal J, Arras G, Colombo M, Jouve M, Morath JP, Primdal-Bengtson B, et al. Proteomic comparison defines novel markers to characterize heterogeneous populations of extracellular vesicle subtypes. Proc Natl Acad Sci. 2016; 113: E968-E77.

26. Jeppesen DK, Fenix AM, Franklin JL, Higginbotham JN, Zhang Q, Zimmerman LJ, et al. Reassessment of Exosome Composition. Cell. 2019; 177: 428-45.e18.

27. Théry C, Witwer KW, Aikawa E, Alcaraz MJ, Anderson JD, Andriantsitohaina $\mathrm{R}$, et al. Minimal information for studies of extracellular vesicles 2018 (MISEV2018): a position statement of the International Society for Extracellular Vesicles and update of the MISEV2014 guidelines. J Extracell Vesicles. 2018; 7: 1535750.

28. Syn NL, Wang L, Chow EK, Lim CT, Goh BC. Exosomes in Cancer Nanomedicine and Immunotherapy: Prospects and Challenges. Trends Biotechnol. 2017; 35: 665-76.

29. Hwang DW. Perspective in Nuclear Theranostics Using Exosome for the Brain. Nucl Med Mol Imaging. 2019; 53: 108-14.

30. Riazifar M, Mohammadi MR, Pone EJ, Yeri A, Lässer C, Segaliny AI, et al. Stem Cell-Derived Exosomes as Nanotherapeutics for Autoimmune and Neurodegenerative Disorders. ACS Nano. 2019; 13: 6670-6688.

31. Peer D, Karp JM, Hong S, Farokhzad OC, Margalit R, Langer R. Nanocarriers as an emerging platform for cancer therapy. Nat Nanotechnology. 2007; 2: 751-760.

32. Berenguer I, Lagerweij $T$, Zhao XW, Dusoswa $S$, van der Stoop P, Westerman $\mathrm{B}$, et al. Glycosylated extracellular vesicles released by glioblastoma cells are decorated by CCL18 allowing for cellular uptake via chemokine receptor CCR8. J Extracell Vesicles. 2018; 7: 1446660.

33. Rana S, Yue S, Stadel D, Zoller M. Toward tailored exosomes: the exosomal tetraspanin web contributes to target cell selection. Int J Biochem Cell Biol. 2012; 44: 1574-84.

34. Tian $\mathrm{T}$, Zhang HX, He CP, Fan $\mathrm{S}$, Zhu YL, Oi C, et al. Surface functionalized exosomes as targeted drug delivery vehicles for cerebral ischemia therapy. Biomaterials. 2018; 150: 137-49.

35. Lin $\mathrm{Y}, \mathrm{Lu} \mathrm{Y}, \mathrm{Li} \mathrm{X}$. Biological characteristics of exosomes and genetically engineered exosomes for the targeted delivery of therapeutic agents. J Drug Target. 2020; 28: 129-41.

36. Walker S, Busatto S, Pham A, Tian M, Suh A, Carson K, et al. Extracellular vesicle-based drug delivery systems for cancer treatment. Theranostics. 2019; 9: 8001-17.
37. Elsharkasy OM, Nordin JZ, Hagey DW, de Jong OG, Schiffelers RM, Andaloussi SE, et al. Extracellular vesicles as drug delivery systems: Why and how? Adv Drug Deliv Rev, in press. 2020; doi: 10.1016/j.addr.2020.04.004.

38. Lai CP, Mardini O, Ericsson M, Prabhakar S, Maguire C, Chen JW, et al. Dynamic biodistribution of extracellular vesicles in vivo using a multimodal imaging reporter. ACS Nano. 2014; 8: 483-94.

39. Lara P, Palma-Florez S, Salas-Huenuleo E, Polakovicova I, Guerrero S, Lobos-Gonzalez L, et al. Gold nanoparticle based double-labeling of melanoma extracellular vesicles to determine the specificity of uptake by cells and preferential accumulation in small metastatic lung tumors. J Nanobiotechnology. 2020; 18: 20.

40. Alvarez-Erviti L, Seow Y, Yin H, Betts C, Lakhal S, Wood MJ. Delivery of siRNA to the mouse brain by systemic injection of targeted exosomes. Nat Biotechnol. 2011; 29: 341-5.

41. Betzer O, Perets N, Angel A, Motiei M, Sadan T, Yadid G, et al. In Vivo Neuroimaging of Exosomes Using Gold Nanoparticles. ACS Nano. 2017; 11: 10883-93.

42. Zhang P, Dong B, Zeng E, Wang F, Jiang Y, Li D, et al. In Vivo Tracking of Multiple Tumor Exosomes Labeled by Phospholipid-Based Bioorthogonal Conjugation. Anal Chem. 2018; 90: 11273-9.

43. Headland SE, Jones HR, Norling LV, Kim A, Souza PR, Corsiero E, et al. Neutrophil-derived microvesicles enter cartilage and protect the joint in inflammatory arthritis. Sci Transl Med. 2015; 7: 315ra190.

44. Anderson MR, Kashanchi F, Jacobson S. Exosomes in Viral Disease. Neurotherapeutics. 2016; 13: 535-46.

45. Kamerkar S, LeBleu VS, Sugimoto H, Yang S, Ruivo CF, Melo SA, et al. Exosomes facilitate therapeutic targeting of oncogenic KRAS in pancreatic cancer. Nature. 2017; 546: 498-503.

46. Millard M, Yakavets I, Piffoux M, Brun A, Gazeau F, Guigner J-M, et al. mTHPC-loaded extracellular vesicles outperform liposomal and free mTHPC formulations by an increased stability, drug delivery efficiency and cytotoxic effect in tridimensional model of tumors. Drug Deliv. 2018; 25: 1790-801.

47. Schindler C, Collinson A, Matthews C, Pointon A, Jenkinson L, Minter RR, et al. Exosomal delivery of doxorubicin enables rapid cell entry and enhanced in vitro potency. PLoS One. 2019; 14: e0214545.

48. Morse MA, Garst J, Osada T, Khan S, Hobeika A, Clay TM, et al. A phase I study of dexosome immunotherapy in patients with advanced non-small cell lung cancer. J Transl Med. 2005; 3: 9 .

49. Escudier B, Dorval T, Chaput N, André F, Caby MP, Novault S, et al. Vaccination of metastatic melanoma patients with autologous dendritic cell (DC) derived-exosomes: results of thefirst phase I clinical trial. J Transl Med. 2005: $3: 10$.

50. Dai S, Wei D, Wu Z, Zhou X, Wei X, Huang H, et al. Phase I clinical trial of autologous ascites-derived exosomes combined with GM-CSF for colorectal cancer. Mol Ther. 2008; 16: 782-90.

51. Besse B, Charrier M, Lapierre V, Dansin E, Lantz O, Planchard D, et al. Dendritic cell-derived exosomes as maintenance immunotherapy after first line chemotherapy in NSCLC. Oncoimmunology. 2016; 5.

52. Nassar W, El-Ansary M, Sabry D, Mostafa MA, Fayad T, Kotb E, et al. Umbilical cord mesenchymal stem cells derived extracellular vesicles can safely ameliorate the progression of chronic kidney diseases. Biomater Res. 2016; 20: 21.

53. Zhang X, Liu J, Yu B, Ma F, Ren X, Li X. Effects of mesenchymal stem cells and their exosomes on the healing of large and refractory macular holes. Graefes Arch Clin Exp Ophthalmol. 2018; 256: 2041-52.

54. Ojha T, Rizzo L, Storm G, Kiessling F, Lammers T. Image-guided drug delivery: preclinical applications and clinical translation. Expert Opin Drug Deliv. 2015; 12: 1203-7.

55. Chuo ST, Chien JC, Lai CP. Imaging extracellular vesicles: current and emerging methods. J Biomed Sci. 2018; 25: 91.

56. James ML, Gambhir SS. A Molecular Imaging Primer: Modalities, Imaging Agents, and Applications. Physiol Rev. 2012; 92: 897-965.

57. Hu L, Wickline SA, Hood JL. Magnetic Resonance Imaging of Melanoma Exosomes in Lymph Nodes. Magn Reson Med. 2014; 74: 266-71

58. Busato A, Bonafede R, Bontempi P, Scambi I, Schiaffino L, Benati D, et al. Magnetic resonance imaging of ultrasmall superparamagnetic iron oxide-labeled exosomes from stem cells: a new method to obtain labeled exosomes. Int J Nanomedicine. 2016; 11: 2481- 90.

59. Busato A, Bonafede R, Bontempi P, Scambi I, Schiaffino L, Benati D, et al. Labeling and Magnetic Resonance Imaging of Exosomes Isolated from Adipose Stem Cells. Curr Protoc Cell Biol. 2017; 75: 3.44.1-3.44.15.

60. Abello J, Nguyen TDT, Marasini R, Aryal S, Weiss ML. Biodistribution of gadolinium- and near infrared-labeled human umbilical cord mesenchymal stromal cell-derived exosomes in tumor bearing mice. Theranostics. 2019; 9: 2325-45.

61. Dabrowska S, Del Fattore A, Karnas E, Frontczak-Baniewicz M, Kozlowska H, Muraca $\mathrm{M}$, et al. Imaging of extracellular vesicles derived from human bone marrow mesenchymal stem cells using fluorescent and magnetic labels. Int J Nanomedicine. 2018; 13: 1653-64.

62. Liu $\mathrm{T}$, Zhu $\mathrm{Y}$, Zhao $\mathrm{R}$, Wei $\mathrm{X}$, Xin $\mathrm{X}$. Visualization of exosomes from mesenchymal stem cells in vivo by magnetic resonance imaging. Magn Reson Imaging. 2020; 68: 75-82.

63. Cherry SR, Sorenson JA, Phelps ME. The Gamma Camera: Basic Principles. In: Physics in Nuclear Medicine, $4^{\text {th }}$ ed. Philadelphia: W.B. Saunders. 2012; p: 195-208. 
64. Shukla AK, Kumar U. Positron emission tomography: An overview. J Med Phys. 2006; 31: 13-21.

65. Rahmim A, Zaidi H. PET versus SPECT: strengths, limitations and challenges. Nucl Med Commun. 2008; 29: 193-207.

66. Shen L-M, Quan L, Liu J. Tracking Exosomes in Vitro and in Vivo To Elucidate Their Physiological Functions: Implications for Diagnostic and Therapeutic Nanocarriers. ACS Appl Nano Mater. 2018; 1: 2438-48.

67. Panagopoulou MS, Wark AW, Birch DJS, Gregory CD. Phenotypic analysis of extracellular vesicles: a review on the applications of fluorescence. J Extracell Vesicles. 2020; 9: 1710020.

68. Antimisiaris SG, Mourtas S, Marazioti A. Exosomes and Exosome-Inspired Vesicles for Targeted Drug Delivery. Pharmaceutics. 2018; 10: 218.

69. Man F, Gawne PJ, T.M. de Rosales R. Nuclear imaging of liposomal drug delivery systems: A critical review of radiolabelling methods and applications in nanomedicine. Adv Drug Deliv Rev. 2019; 143: 134-60.

70. Hu Q, Su H, Li J, Lyon C, Tang W, Wan M, et al. Clinical applications of exosome membrane proteins. Precis Clin Med. 2020; 3: 54-66.

71. Skotland T, Sagini K, Sandvig K, Llorente A. An emerging focus on lipids in extracellular vesicles. Adv Drug Deliv Rev, in press. 2020; doi: 10.1016/j.addr.2020.03.002.

72. Clayton A, Harris CL, Court J, Mason MD, Morgan BP. Antigen-presenting cell exosomes are protected from complement-mediated lysis by expression of CD55 and CD59. Eur J Immunol. 2003; 33: 522-31.

73. Clayton A, Turkes A, Dewitt S, Steadman R, Mason MD, Hallett MB. Adhesion and signaling by B cell-derived exosomes: the role of integrins. The FASEB Journal. 2004; 18: 977-9.

74. Hoshino A, Costa-Silva B, Shen T-L, Rodrigues G, Hashimoto A, Tesic Mark $\mathrm{M}$, et al. Tumour exosome integrins determine organotropic metastasis. Nature. 2015; 527: 329-35

75. Rayamajhi S, Aryal S. Surface functionalization strategies of extracellular vesicles. J Mater Chem B. 2020; 8: 4552-69.

76. Phillips WT, Rudolph AS, Goins B, Timmons JH, Klipper R, Blumhardt R. A simple method for producing a technetium-99m-labeled liposome which is stable In Vivo. Int J Rad Appl Instrum B. 1992; 19: 539-47.

77. Edmonds S, Volpe A, Shmeeda H, Parente-Pereira AC, Radia R, Baguna-Torres J, et al. Exploiting the Metal-Chelating Properties of the Drug Cargo for In Vivo Positron Emission Tomography Imaging of Liposomal Nanomedicines. ACS Nano. 2016; 10: 10294-307.

78. Morishita M, Takahashi Y, Nishikawa M, Sano K, Kato K, Yamashita T, et al. Quantitative Analysis of Tissue Distribution of the B16BL6-Derived Exosomes Using a Streptavidin-Lactadherin Fusion Protein and Iodine-125-Labeled Biotin Derivative After Intravenous Injection in Mice. J Pharm Sci. 2015; 104: 705-13.

79. Matsumoto A, Takahashi Y, Nishikawa M, Sano K, Morishita M, Charoenviriyakul C, et al. Accelerated growth of B16BL6 tumor in mice through efficient uptake of their own exosomes by B16BL6 cells. Cancer Sci. 2017; 108: 1803-10.

80. Rashid MH, Borin TF, Ara R, Angara K, Cai J, Achyut BR, et al. Differential in vivo biodistribution of 131I-labeled exosomes from diverse cellular origins and its implication for theranostic application. Nanomedicine. 2019; 21: 102072.

81. Varga Z, Gyurko I, Paloczi K, Buzas EI, Horvath I, Hegedus N, et al. Radiolabeling of Extracellular Vesicles with $(99 \mathrm{~m}) \mathrm{Tc}$ for Quantitative In Vivo Imaging Studies. Cancer Biother Radiopharm. 2016; 31: 168-73.

82. Molavipordanjani S, Khodashenas S, Abedi SM, Moghadam MF, Mardanshahi A, Hosseinimehr SJ. 99mTc-radiolabeled HER2 targeted exosome for tumor imaging. Eur J Pharm Sci. 2020; 148: 105312.

83. González MI, Martín-Duque P, Desco M, Salinas B. Radioactive Labeling of Milk-Derived Exosomes with 99mTc and In Vivo Tracking by SPECT Imaging. Nanomaterials. 2020; 10: 1062.

84. Faruqu FN, Wang JT, Xu L, McNickle L, Chong EM, Walters A, et al. Membrane Radiolabelling of Exosomes for Comparative Biodistribution Analysis in Immunocompetent and Immunodeficient Mice - A Novel and Universal Approach. Theranostics. 2019; 9: 1666-82.

85. Smyth T, Kullberg M, Malik N, Smith-Jones P, Graner MW, Anchordoquy TJ. Biodistribution and Delivery Efficiency of Unmodified Tumor-Derived Exosomes. J Control Release. 2015; 199: 145-55.

86. Rashid MH, Borin TF, Ara R, Alptekin A, Liu YT, Arbab AS. Generation of Novel Diagnostic and Therapeutic Exosomes to Detect and Deplete Protumorigenic M2 Macrophages. Adv Therap. 2020; 3: 12.

87. Hwang DW, Choi H, Jang SC, Yoo MY, Park JY, Choi NE, et al. Noninvasive imaging of radiolabeled exosome-mimetic nanovesicle using (99m)Tc-HMPAO. Sci Rep. 2015; 5: 15636.

88. Gangadaran P, Hong CM, Oh JM, Rajendran RL, Kalimuthu S, Son SH, et al. In vivo Non-invasive Imaging of Radio-Labeled Exosome-Mimetics Derived From Red Blood Cells in Mice. Front Pharmacol. 2018; 9: 817.

89. Royo F, Cossío U, Ruiz de Angulo A, Llop J, Falcon-Perez JM. Modification of the glycosylation of extracellular vesicles alters their biodistribution in mice. Nanoscale. 2019; 11: 1531-7.

90. Banerjee A, Alves V, Rondão T, Sereno J, Neves Â, Lino M, et al. A positron-emission tomography (PET)/magnetic resonance imaging (MRI) platform to track in vivo small extracellular vesicles. Nanoscale. 2019; 11: 13243-8.

91. Shi S, Li T, Wen X, Wu SY, Xiong C, Zhao J, et al. Copper-64 Labeled PEGylated Exosomes for In Vivo Positron Emission Tomography and Enhanced Tumor Retention. Bioconjug Chem. 2019; 30: 2675-83.
92. Jung KO, Kim Y-H, Chung S-J, Kang KW, Rhee S, Pratx G, et al. Highly Sensitive Identification of Lymphatic and Hematogenous Metastasis Routes of Novel Radiolabeled Exosomes Using Non-invasive PET Imaging. bioRxiv. 2020. doi: 10.1101/2020.03.17.995860.

93. Khan A, Man F, Faruqu F, Kim J, Al-Salemee F, Volpe A, et al. [89Zr]Zr(oxinate)4 Allows Direct Radiolabelling and PET Imaging of Small Extracellular Vesicles. ChemRxiv. 2020. doi: 10.26434/chemrxiv.12730463.v1

94. Boerman OC, van Schaijk FG, Oyen WJ, Corstens FH. Pretargeted radioimmunotherapy of cancer: progress step by step. J Nucl Med. 2003; 44: 400-11.

95. Saha GB. Radiopharmaceuticals and General Methods of Radiolabelling. Fundamentals of Nuclear Pharmacy. 7th ed. New York, United States: Springer; 2017; p. 93-121.

96. Russell J, O'Donoghue JA, Finn R, Koziorowski J, Ruan S, Humm JL, et al. Iodination of Annexin V for Imaging Apoptosis. J Nucl Med. 2002; 43: 671-7.

97. Varga Z, Szigyártó IC, Gyurkó I, Dóczi R, Horváth I, Máthé D, et al. Radiolabeling and Quantitative In Vivo SPECT/CT Imaging Study of Liposomes Using the Novel Iminothiolane-99mTc-Tricarbonyl Complex. Contrast Media Mol Imaging. 2017; 2017: 4693417.

98. Tait JF, Smith C, Gibson DF. Development of Annexin V Mutants Suitable for Labeling with Tc(I)-Carbonyl Complex. Bioconjug Chem. 2002; 13: 1119-23.

99. Alberto R. $[\mathrm{Tc}(\mathrm{CO}) 3]+$ chemistry: a promising new concept for SPET? Eur J Nucl Med Mol Imaging. 2003; 30: 1299-302.

100. Srivastava SC, Rao Chervu L. Radionuclide-labeled red blood cells: Current status and future prospects. Semin Nucl Med. 1984; 14: 68-82.

101. Gunter KP, Lukens JN, Clanton JA, Morris PJ, Janco RL, English D. Neutrophil labeling with indium-111: tropolone vs. oxine. Radiology. 1983; 149: 563-6.

102. Costa J. Glycoconjugates from extracellular vesicles: Structures, functions and emerging potential as cancer biomarkers. Biochim Biophys Acta Rev Cancer. 2017; 1868: 157-66.

103. Williams C, Royo F, Aizpurua-Olaizola O, Pazos R, Boons G-J, Reichardt N-C, et al. Glycosylation of extracellular vesicles: current knowledge, tools and clinical perspectives. J Extracell Vesicles. 2018; 7: 1442985.

104. Saunderson SC, Dunn AC, Crocker PR, McLellan AD. CD169 mediates the capture of exosomes in spleen and lymph node. Blood. 2014; 123: 208-16.

105. Morishita M, Takahashi Y, Nishikawa M, Takakura Y. Pharmacokinetics of Exosomes-An Important Factor for Elucidating the Biological Roles of Exosomes and for the Development of Exosome-Based Therapeutics. J Pharm Sci. 2017; 106: 2265-9.

106. Wiklander OPB, Nordin JZ, O'Loughlin A, Gustafsson Y, Corso G, Mäger I, et al. Extracellular vesicle in vivo biodistribution is determined by cell source, route of administration and targeting. J Extracell Vesicles. 2015; 4.

107. Jokerst JV, Lobovkina T, Zare RN, Gambhir SS. Nanoparticle PEGylation for imaging and therapy. Nanomedicine (Lond). 2011; 6: 715-28.

108. Emam SE, Abu Lila AS, Elsadek NE, Ando $\mathrm{H}$, Shimizu $\mathrm{T}$, Okuhira $\mathrm{K}$, et al. Cancer cell-type tropism is one of crucial determinants for the efficient systemic delivery of cancer cell-derived exosomes to tumor tissues. Eur J Pharm Biopharm. 2019; 145: 27-34.

109. Cooper MS, Ma MT, Sunassee K, Shaw KP, Williams JD, Paul RL, et al. Comparison of (64)Cu-complexing bifunctional chelators for radioimmunoconjugation: labeling efficiency, specific activity, and in vitro/in vivo stability. Bioconjug Chem. 2012; 23: 1029-39.

110. Wu N, Kang CS, Sin I, Ren S, Liu D, Ruthengael VC, et al. Promising bifunctional chelators for copper 64-PET imaging: practical $(64) \mathrm{Cu}$ radiolabeling and high in vitro and in vivo complex stability. J Biol Inorg Chem. 2016; 21: 177-84

111. Man F, Lim L, Volpe A, Gabizon A, Shmeeda H, Draper B, et al. In Vivo PET Tracking of 89Zr-Labeled Vy9V82 T Cells to Mouse Xenograft Breast Tumors Activated with Liposomal Alendronate. Mol Ther. 2019; 27: 219-29.

112. Gawne PJ, Clarke F, Turjeman K, Cope AP, Long NJ, Barenholz Y, et al. PET Imaging of Liposomal Glucocorticoids using < sup $>89</$ sup $>\mathrm{Zr}$-oxine: Theranostic Applications in Inflammatory Arthritis. Theranostics. 2020; 10: 3867-79.

113. Jang SC, Kim OY, Yoon CM, Choi D-S, Roh T-Y, Park J, et al. Bioinspired Exosome-Mimetic Nanovesicles for Targeted Delivery of Chemotherapeutics to Malignant Tumors. ACS Nano. 2013; 7: 7698-710.

114. Saha GB. Characteristics of Specific Radiopharmaceuticals. Fundamentals of Nuclear Pharmacy. 6th ed. New York, United States: Springer; 2010; p. 115-52.

115. Son $\mathrm{SH}, \mathrm{Oh}$ JM, Gangadaran P, Ji HD, Lee HW, Rajendran RL, et al. White blood cell labeling with Technetium-99m (99mTc) using red blood cell extracellular vesicles-mimetics. Blood Cells Mol Dis. 2020; 80: 102375.

116. Takahashi $Y$, Nishikawa $M$, Shinotsuka $H$, Matsui $Y$, Ohara $S$, Imai $T$, et al. Visualization and in vivo tracking of the exosomes of murine melanoma B16-BL6 cells in mice after intravenous injection. J Biotechnol. 2013; 165: 77-84.

117. Choi HS, Liu W, Misra P, Tanaka E, Zimmer JP, Itty Ipe B, et al. Renal clearance of quantum dots. Nat Biotechnol. 2007; 25: 1165-70.

118. Lobo ED, Hansen RJ, Balthasar JP. Antibody Pharmacokinetics and Pharmacodynamics. J Pharm Sci. 2004; 93: 2645-68.

119. Chapman AP, Antoniw P, Spitali M, West S, Stephens S, King DJ. Therapeutic antibody fragments with prolonged in vivo half-lives. Nat Biotechnol. 1999; 17: 780-3.

120. Baranyai T, Herczeg K, Onódi Z, Voszka I, Módos K, Marton N, et al. Isolation of Exosomes from Blood Plasma: Qualitative and Quantitative Comparison of Ultracentrifugation and Size Exclusion Chromatography Methods. PLoS One. 2015; 10: e0145686-e. 
121. Webber J, Clayton A. How pure are your vesicles? J Extracell Vesicles. 2013; 2.

122. Takov K, Yellon DM, Davidson SM. Comparison of small extracellular vesicles isolated from plasma by ultracentrifugation or size-exclusion chromatography: yield, purity and functional potential. J Extracell Vesicles. 2019; 8: 1560809 .

123. Wei R, Zhao L, Kong G, Liu X, Zhu S, Zhang S, et al. Combination of Size-Exclusion Chromatography and Ultracentrifugation Improves the Proteomic Profiling of Plasma-Derived Small Extracellular Vesicles. Biol Proced Online. 2020; 22: 12.

124. Willms E, Johansson HJ, Mäger I, Lee Y, Blomberg KEM, Sadik M, et al. Cells release subpopulations of exosomes with distinct molecular and biological properties. Sci Rep. 2016; 6: 22519.

125. Zabeo D, Cvjetkovic A, Lässer C, Schorb M, Lötvall J, Höög JL. Exosomes purified from a single cell type have diverse morphology. J Extracell Vesicles. 2017; 6: 1329476 